\title{
CANONICAL NEIGHBORHOODS FOR TOPOLOGICALLY EMBEDDED POLYHEDRA
}

\author{
BY \\ ROBERT CRAGGS( ${ }^{1}$ )
}

\begin{abstract}
D. R. McMillan has shown that in any neighborhood of a compact two sided surface in a 3-manifold there is a closed neighborhood of the surface which is the sum of a solid homeomorphic to the cartesian product of the surface with the unit interval and some small disjoint cubes-with-handles each of which intersects the cartesian product in a disk on its boundary. In the present paper the author generalizes this notion of canonical neighborhood so that it applies to topological embeddings of arbitrary polyhedra in 3 -manifolds. This is done by replacing the cartesian products by small regular neighborhoods of polyhedral approximations to the topological embeddings.
\end{abstract}

In [20] McMillan established the following result on neighborhoods of surfaces in 3-manifolds:

Theorem. Suppose $S$ is a compact, boundaryless, polybedral surface, $M$ is a pwl 3-manifold, and $f$ is a bomeomorphis $m$ of $S$ on to a two sided surface in Int $M$. Then for each $\epsilon>0$ there is a pwl embedding $g$ of $S \times[0,1]$ into Int $M$ and there is a finite collection $\left\{H_{i}\right\}$ of mutually exclusive, polybedral cubes-with. bandles in Int $M$ such that

(1) for eacb $y \in S, \rho(f(y), g(y, e))<\epsilon(e=0,1)$,

(2) each dia $H_{i}<\epsilon$ and eacb $H_{i} \cap g(S \times[0,1])$ is a disk in $\mathrm{Bd} H_{i}$, and

(3) $g(S \times[0,1]) \cup \cup H_{i}$ contains a neigbborbood of $f(S)$ in $M$.

In this paper we generalize McMillan's notion of a canonical neighborhood so that it applies to embeddings of arbitrary polyhedra in 3-manifolds. In our system regular neighborhoods of approximating polyhedra take the place of embeddings of cartesian products. In $\$ 8$ we obtain existence and uniqueness the orems for canonical neighborhoods. In $\$ 9$ we sharpen McMillan's theorem for surfaces by re moving the compactness and no boundary assumptions and by showing that the diameters of the fibers of $g(S \times[0,1])$ can be kept small.

Notation. Some of our conventions are taken from [8], [9], [10]; however,

Presented to the Society, December 15, 1966 under the title Neighborhoods of polyhedra in 3-manifolds; received by the editors April 29, 1971.

AMS (MOS) subject clas sifications (1970). Primary 55A30, 57A 10; Secondary 57C35, $57 \mathrm{C} 40$.

Key words and phrases. 3-manifold, neighborhoods of surfaces, canonical neighborhoods, topological embeddings of polyhedra, small regular neighborhoods, approximating polyhedra, cubes-with-handles.

(1) Research supported in part by NSF contracts GP-3857 and GP-5804. 
we have changed our use of several terms in this paper.

Manifold and manifold-with-boundary are taken as synonymous. A surface is a 2-manifold. If $Y \subseteq X$ then $\operatorname{Fr} Y$ denotes the set $(\mathrm{Cl} Y) \cap \mathrm{Cl}(X-Y)$. If we write that a function, say $f$, takes a pair $(X, Y)$ into a pair $(U, V)$ then we mean that $f^{-1}(U)=X$ and $f^{-1}(V)=Y$. We use $\rho$ to denote a metric and $d(Y, Z)$ to denote the distance between sets $Y$ and $Z$.

We use $C(X)$ to denote the set of continuous, nonnegative functions $\mu$ on $X$ such that, for each $t>0, \mu^{-1}([t, \infty))$ is compact. We use $C(X, Y)$ and $C(X, Y, Z)$ to denote respectively the subset of $C(X)$ consisting of the functions which are positive on $Y$ and the subset of $C(X)$ consisting of the functions which are positive on $Y$ and 0 on $Z$. We write $\mu_{1} \leq \mu_{2}$ if $\mu_{1}(x) \leq \mu_{2}(x)$ for each $x$ in the common doma in of $\mu_{1}$ and $\mu_{2}$. Let $\mu \in C(X)$. A map $f$ of a subset $Y$ of $X$ into $X$ is a $\mu$-map if, for each $y \in Y, \rho(y, f(y)) \leq \mu(y)$. If $f$ and $g$ are maps of $Y$ into $X$, then $g$ is a $\mu$-approximation to $f$ provided $g f^{-1}$ is a $\mu$-map of $f(Y)$. A subset $Z \subseteq X$ is a $\mu$-set if $Z$ is contained in an open set $O(Z)$ with dia $O(Z) \leq \inf \mu$ on $O(Z)$.

For $\mu_{1}$ and $\mu_{2} \in C(X)$ and a natural number $k$, we say that $\left(X, \mu_{1}, \mu_{2}, k\right)$ has Property $S$ if, for every sequence of points $x_{1}, \cdots, x_{k}$ such that each $\rho\left(x_{i}, x_{i+1}\right) \leq$ $\mu_{2}\left(x_{i}\right), \mu_{2}\left(x_{k}\right) \leq \mu_{1}\left(x_{1}\right)$ and $\mu_{1}\left(x_{1}\right) \leq 2 \mu_{1}\left(x_{k}\right)$. The composition of $k \mu$-maps is not necessarily a $(k \mu)$-map, and the inverse of a $\mu$-map is not necessarily a $\mu$-map. The two conditions in the definition of Property $S$ are used to get around these two facts.

We follow unpublished notes of M. H. A. Newman in using $\theta\left(\begin{array}{l}a_{1} \cdots a_{n} \\ b_{1} \cdots b_{n}\end{array}\right)$ to denote the map of $\left[a_{1}, a_{n}\right]$ onto $\left[b_{1}, b_{n}\right]$ which takes each $a_{i}$ onto $b_{i}$ and is affine on each subinterval $\left[a_{i}, a_{i+1}\right]$. If $Y \subseteq X$ then a collar on $Y$ in $X$ is a closed embed$\operatorname{ding} \phi: Y \times[0, r] \rightarrow X$ for some $r \neq 0$ such that, for each $y \in Y, \phi(y, 0)=y$ and $\phi(Y \times[0, r])$ contains a neighborhood of $Y$. If $X$ and $Y$ are polyhedra then $\phi$ is a pwl collar provided the map $\phi$ is pwl. We say that $\phi$ is a proper collar provided that any homeomorphism $b$ of $Y \times[0, r]$ onto itself which takes each fiber $y \times[0, r]$ onto itself and is the identity on $Y \times\{0, r\}$ induces a homeomorphism of $X$ which is an extension of $\phi b \phi^{-1}$. If $\mu \in C(X)$ then $\phi$ is a $\mu$-collar provided each $\phi(y \times[0, r])$ is a $\mu$-set.

We emphasize that here polyhedra are not necessarily compact. We write $<$ to indicate a subcomplex or subpolyhedron, and we use $\checkmark$ to indicate a full subcomplex. For a complex $K,|K|$ denotes the carrier of $K$, and if $X$ is a set in $|K|$ then $[X]$ denotes the intersection of all subcomplexes of $K$ whose carriers contain $X$, and $N(X, K)$ denotes the polyhedron underlying the intersection of all subcomplexes of $K$ whose carriers contain neighborhoods of $X$. For a simplex $s$ in a complex $K, \mathrm{lk}(s, K)=\{t: s * t \in K\}$, st $(s, K)=s * \mathrm{k}(s, K)$, and $\mathrm{st}^{\circ}(s, K)=|\mathrm{st}(s, K)|-$ $|\dot{s} * \mathrm{lk}(s, K)|$. For a complex $K, K_{i}$ denotes the $i$-skeleton of $K$. For a simplex $s$ 
of $K, b(s)$ denotes the barycenter of $s$, or if we are dealing with a derived subdivision of $K, d(s)$ denotes the vertex in Int $s$. Here $K^{n}$ denotes an $n$th derived subdivision of $K$, and for $L<K,(K, L)^{n}$ denotes an $n$th derived subdivision of $K$ modulo $L$. In $(K, L)^{1}$ there is one vertex in Int $s$ for each simplex $s$ of $K-L$.

The following definitions on collapsing, although somewhat restrictive, are sufficient for our needs. First $\succ^{s}, \searrow^{e}$, and $\succ^{c}$ denote respectively simplicial collapse, elementary collapse, and collection of disjoint collapses with compact supports. We explain this last term more fully: $X\rangle^{c} Y$ means that $X$ and $Y$ are polyhedra with $Y \subset X, \mathrm{Cl}(X-Y)$ is a polyhedron, and if $C$ is a component of $\mathrm{Cl}(X-Y)$ then $C$ is compact and $C \searrow C \cap Y$. Finally, for polyhedra $X$ and $Y$ which are not necessarily compact, $X \searrow Y$ means that there is a finite sequence of collapses $X=X_{0} \searrow^{c} X_{1} \searrow^{c} \ldots \searrow^{c} X_{k}=Y$. For polyhedra $X, Y$, and $Z$, an elementary collapse $X \searrow^{e} Y$ is admissible with respect to $Z$ if $(X-Y) \cap Z \neq \emptyset$ implies that $X-Y \subseteq Z$. Similarly, a collapse $X \searrow^{c} Y$ is admissible with respect to $Z$ if for each component $C$ of $\mathrm{Cl}(X-Y)$, the collapse $C \searrow C \cap Y$ can be made by way of a finite sequence of elementary collapses which are admissible with respect to $Z$. Admissibility of arbitrary collapses is defined in the obvious fashion. For $X \subseteq M$ and $\mu \in C(M)$, we say that a collapse $X \searrow Y$ is a $\mu$-collapse if each component of $\mathrm{Cl}(X-Y)$ is a compact $\mu$-set. We write $X \searrow 0$ if $X$ collapses to a point.

A triangulation of a polyhedron $P$ is a pair $(J, \phi)$ consisting of a rectilinear simplicial complex $J$ in some Euclidean space together with a pwl homeomorphism $\phi$ of $|J|$ onto $P$. If $P(1), \cdots, P(k)$ are subpolyhedra of $P$ then $(J, J(1), \cdots$, $J(k), \phi)$ triangulates $(P, P(1), \cdots, P(k))$ if each $J(i)<J$ and $\phi(|J(i)|)=P(i)$.

We follow Cohen [7] in defining regular neigbborbood as follows: If $P<Q$ $<R$ are polyhedra (not necessarily compact) then $Q$ is a regular neighborhood of $P$ in $R$ if there is a triangulation $(J, L, \phi)$ of $(R, P)$ in which $L \triangleleft J$ and there is a first derived subdivision $J^{1}$ of $J$ such that $Q=\phi\left(N\left(L^{1}, J^{1}\right)\right)$. If $\mu \in C(R)$ then $Q$ is a $\mu$-regular neigbborbood of $P$ in $R$ if, for some triangulation $(J, L, \phi)$ as above, $\phi(N(t, J))$ is a $\mu$-set for each $t \in L$.

Suppose $P$ is a polyhedron, $P_{a}<P$, and $f$ is a closed embedding of $\left(P, P_{a}\right)$ into $(M, \mathrm{Bd} M)$. Suppose $\mu \in C(M, f(P))$. A canonical neigbborbood system for $(M, P, f, \mu)$ is a triple $\left(g, N,\left\{H_{i}\right\}\right)$ consisting of a pwl homeomorphism $g:(P, P a)$ $\rightarrow(M, \mathrm{Bd} M)$ which $\mu$-approximates $f$, a $\mu$-regular neighborhood $N$ of $g(P)$ in $M$, and a discrete collection of polyhedral cubes-with-handles $\left\{H_{i}\right\}$ in $M$ such that each $H_{i}$ is a $\mu$-set in Int $M$ which intersects $N$ in a disk and $N \cup \cup H_{i}$ contains a neighborhood of $f(P)$ in $M$. The polyhedron $N \cup \cup H_{i}$ will be called a canonical neighborbood for $(M, P, f, \mu)$.

Next we describe a notion of reduction of a complex. Reduction is used to alter a complex so that it becomes more like a combinatorial 3-manifold. We begin 
with complexes $K_{a} \triangleleft K$ and $L_{a} \triangleleft L$ and a pwl map $r: L \rightarrow K$ such that $r\left(L_{a}\right)=$ $K_{a}$.

We say that $\left(L, L_{a}, r\right)$ reduces $\left(K, K_{a}\right)$ at a set of 2-simplexes $\left\{\Delta_{i}\right\}$ of $K$ provided (1) $K<L$ and $K_{a}=L_{a}$, (2) each $\operatorname{lk}\left(\Delta_{i}, K\right)=\varnothing$ and each $1 \mathrm{k}\left(\Delta_{i}, L\right)$ is a vertex $w_{i}$ in $L_{0}-K_{0}$ where $w_{i} \neq w_{j}$ for $i \neq j$, (3) $L=K \cup \cup w_{i} * \Delta_{i}$, and (4) $r$ is a retraction which is affine on each simplex of $L$ and sends each $w_{i}$ to $b\left(\Delta_{i}\right)$.

We say that $\left(L, L_{a}, r\right)$ is an elementary reduction of $\left(K, K_{a}\right)$ at a pair $(\sigma, S)$ where $\sigma$ is a 1 -simplex of $K$ if (1) $\mathrm{lk}(\sigma, K)$ is a 1 -manifold and $S$ is a 0 -sphere in $\mathrm{l} k(\sigma, K)-K_{a}$ which does not bound in $1 \mathrm{k}(\sigma, K)$, (2) $L=K \cup w * \sigma * S$ where $w$ is a vertex in $L_{0}-K_{0}$ and $L_{a}=K_{a}$, and (3) $r$ is a retraction which is affine on each simplex of $L$ and sends $w$ to $b(\sigma)$.

We say that $\left(L, L_{a}, r\right)$ is an elementary reduction of $\left(K, K_{a}\right)$ at a pair $(\nu, J)$ where $v$ is a vertex of $K$ and $J$ is a 1-sphere in $1 \mathrm{k}(v, K)$ provided (1) each component of $\mathrm{lk}(\nu, K)$ is either a punctured 2-sphere or a point, and $1 \mathrm{k}(\nu, K) \cap K_{a}=$ $\emptyset$ unless $v \in K_{a}$ in which case $\mathrm{lk}\left(v, K_{a}\right)$ is a 1 -sphere in the boundary of some component of $\mathrm{lk}(v, K),(2) \mathrm{lk}(\nu, K)$ is the sum of disjoint complexes $E$ and $F$ where $J$ is in the boundary of some component of $E$ and $J \cap K_{a}=\emptyset$, (4) $L=$ $\{t \in K: v \notin t\} \cup v * E \cup u * F \cup v * u * J$ where $u \in L_{0}-K_{0}$ and $L_{a}=K_{a}$, and (3) $r$ is a simplicial map from $L$ to $K$ which sends $u$ to $v$ and leaves all other vertices fixed. Figure 1.1 illustrates an elementary reduction for a vertex.
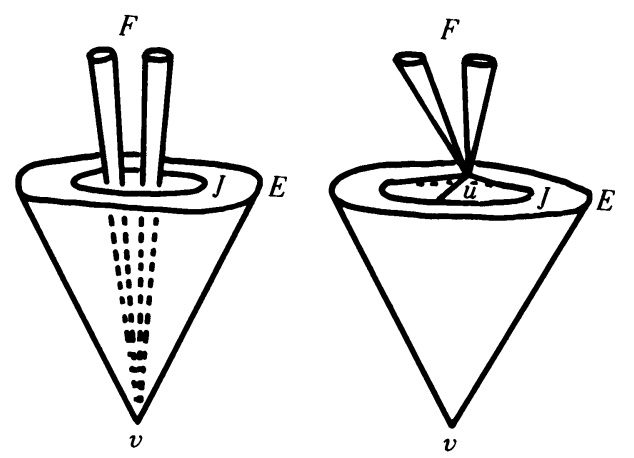

Figure 1.1

We say that $\left(L, L_{a}, r\right)$ reduces $\left(K, K_{a}\right)$ at a set of simplexes $\left\{t_{k}\right\}$ of $K$ where the $t_{k}$ 's are either all 1 -simplexes or all vertices if (1) the complexes $1 \mathrm{k}\left(t_{k}, K\right)$ are mutually exclusive, (2) $r$ is the identity on $|L|-\bigcup_{r^{-1}}\left(s t^{\circ}\left(t_{k}, K\right)\right)$, (3) for each $t_{k}$ there is a sequence of complexes (st $\left(t_{k}, K\right)$, st $\left.\left(t_{k}, K\right) \cap K_{a}\right)=(L(k, 0)$, $\left.L_{a}(k, 0)\right), \cdots,\left(L(k, m), L_{a}(k, m)\right)=\left(r^{-1}\left(\operatorname{st}\left(t_{k}, K\right)\right), r^{-1}\left(\right.\right.$ st $\left.\left.\left(t_{k}, K\right) \cap K_{a}\right)\right)$, there is a sequence $r_{k 1}, \cdots, r_{k m}$ of pwl maps $r_{k i}: L(k, i) \rightarrow L(k, i-1)$ such that $r \mid L(k, m)=r_{k 1} \cdots r_{k m}$ and each $\left(L(k, i), L_{a}(k, i), r_{k i}\right)$ is an elementary reduction of $\left(L(k, i-1), L_{a}(k, i-1)\right)$ at a pair $(t(k, i), S(k, i))$ where $S(k, i)<\mathrm{lk}_{(}\left(t_{k}, K\right)$ 
and $r_{k 1} \cdots r_{k i}(t(k, i))=t_{k}$, and (4) if the $t_{k}$ 's are 1-simplexes then each $\mathrm{lk}\left(t_{k}, L\right)$ is a 1 -ball and if the $t_{k}$ 's are vertices then for each vertex $w$ in $r^{-1}\left(\bigcup_{t_{k}}\right)$, every nondegenerate component of $1 \mathrm{k}(w, L)$ is a disk.

Finally let $P$ and $Q$ be polyhedra, $r$ a pwl map from $Q$ to $P, M$ a pwl 3manifold, $f$ and $g$ closed embeddings of $P$ and $Q$ respectively into $M$, and $\mu \epsilon$ $C(M)$. A special map for $(M, f, g, r, \mu)$ is a continuous $\mu$-map $\pi$ of $M$ onto itself such that

(1) $\pi g=f r$,

(2) $\pi$ takes $(M-g(Q))$ homeomorphically onto $M-f(P)$, and

(3) for each $\mu_{1} \in C\left(M, \mu^{-1}(0, \infty)\right)$ there is a homeomorphism $H$ of $M$ onto itself which $\mu_{1}$-approximates $\pi$.

\section{Some lemmas on Property S.}

Lemma 2.1. Suppose $X$ is a locally compact metric space, $\mu \in C(X)$, and $k$ is a natural number.

Then there is a function $\mu_{1} \in C\left(X, \mu^{-1}(0, \infty)\right)$ such that $\left(X, \mu, \mu_{1}, k\right)$ bas Property S.

Proof. By taking infimums over functions defined for a locally finite collection of compact sets we can reduce the problem to the case where $X$ is compact. For this case define $\mu_{2}$ by

$$
\mu_{2}(x)=\inf \left(\{1\} \cup\left\{\rho(x, y): y \notin \mu^{-1}\left(2^{-1 / k} \mu(x), 2^{1 / k} \mu(x)\right)\right\}\right) .
$$

The compactness of $X$ can be used to show that $\mu_{2}$ is lower semicontinuous. By the Baire insertion theorem (see [14, p. 171]) there is a function $\mu_{3} \in C\left(X, \mu^{-1}(0, \infty)\right)$ with $\mu_{3} \leq \mu_{2}$. Define $\mu_{1}$ by $\mu_{1}(x)=\operatorname{minimum}\left(\mu_{3}(x), \mu(x) / 2\right)$. It is easily verified that $\left(X, \mu, \mu_{1}, k\right)$ has Property S.

Calculations involving Property $S$ will almost always implicitly involve one of the next two lemmas which follow immediately from the definition of Property $\mathrm{S}$.

Lemma 2.2. Suppose $\left(X, \mu, \mu_{1}, k+1\right)$ bas Property $\mathrm{S}$ and suppose $Y \subseteq X$.

Then if $g$ is a $\mu_{1}$-homeomorphism of $Y$ into $X, g^{-1}$ is a $2 \mu$-homeomorphism, and if $b_{1}, \cdots, b_{k}$ are $\mu_{1}$-maps of sets $Y=Y_{1}, \cdots, Y_{k}$ where each $b_{i-1}\left(Y_{i-1}\right)$ $\subseteq Y_{i}$, then $b=b_{k} \cdots b_{1}$ is a $(k \mu)$-map of $Y$ into $X$.

Lemma 2.3. Suppose $S$ is a metrir space and $\mu$ and $\mu_{1} \in C(X)$ are such that $\left(X, \mu / 6, \mu_{1}, 2\right)$ bas Property S.

Then if $Y$ is a $\mu_{1}$-set in $X$ and $f$ is a $\mu_{1}$-map of $Y$ into $X, f(Y)$ is a $\mu$-set.

Lemma 2.4. Suppose $M$ is a (pwl) n-manifold, $S$ is a locally flat $(p w l)(n-1)$ manifold in $\mathrm{Bd} M$ which is a closed subset of $\mathrm{Bd} M$, and $\mu \in C(M, S)$.

Then for any $r \neq 0$ there is a proper $(p w l) \mu$-collar $\phi: S \times[0, r] \rightarrow M$ on $S$ in $M$. 
Proof. The topological version is obtained from Brown's collaring theorem [6] by cutting down the lengths of fibers. It follows from invariance of domain that the fibers can be cut back enough so that the collar is proper. The pwl case follows similarly from $[7,4.2]$.

3. On $\mu$-regular neighborhoods. Here we use Property $\mathrm{S}$ to help identify $\mu$ regular neighborhoods.

Lemma 3.1. Suppose that $M$ is a pwl manifold, $P$ is a polybedron in $M$, and $\mu, \mu_{1}$, and $\mu_{2} \in C(M)$ are such that $\left(M, \mu / 2,2 \mu_{1}, 3\right)$ and $\left(M, \mu_{1}, \mu_{2}, 2\right)$ bave Property $\mathrm{S}$.

Then if $N_{1}$ and $N_{2}$ are $\mu_{2}$-regular neighborboods of $P$ in $M$, there is a pwl $\mu$-bomeomorphism $b$ of $M$ onto itself which is the identity on a polybedral neighborbood of $P$ so that $b\left(N_{1}\right)=N_{2}$.

Proof. Without the $\mu$ 's this lemma is a standard result in regular neighborhood theory. (See [7, 3.1 and 3.2] for example.) In the proof a third regular neighborhood $N_{3}$ of $P$ is constructed and pwl homeomorphisms $b_{1}$ and $b_{2}$ of $M$ onto itself are defined which are the identity on polyhedral neighborhoods of $P$ and take $N_{1}$ and $N_{2}$ onto $N_{3}$. If $\left(J_{1}, L_{1}, \phi_{1}\right)$ and $\left(J_{2}, L_{2}, \phi_{2}\right)$ are the triangulations of $(M, P)$ providing the $\mu_{2}$-structures for $N_{1}$ and $N_{2}$ then we can suppose that for each $i$ and each $t \in J_{i}, b_{i}$ takes $\phi_{i}(t)$ onto itself and $b_{i}$ is the identity on $\phi_{i}(t)$ if $t \cap L_{i}=\emptyset$; thus we can suppose that each $b_{i}$ is a $\mu_{2}$-map. But then by Lemma 2.2 each of $b_{1}$ and $b_{2}^{-1}$ is a $2 \mu_{1}$-map and so $b=b_{2}^{-1} b_{1}$ is a pwl $\mu$-homeomorphism of $M$ onto itself which takes $N_{1}$ onto $N_{2}$ and is the identity on a polyhedral neighborhood of $P$.

Lemma 3.2. Suppose $M$ is a pwl manifold, $P$ is a polybedron in $M$, and $\mu$ and $\mu_{1} \in C(M)$ are such that $\left(M, \mu / 6, \mu_{1}, 2\right)$ bas Property S.

If $N$ is a $\mu_{1}$-regular neighborbood of $P$ in $M$ and $b$ is a pwl $\mu_{1}$-bomeomorphism of $M$ onto itself which is the identity on $P$, then $h(N)$ is a $\mu$-regular neigh. borbood of $P$ in $M$.

Proof. This follows directly from Lemma 2.3.

Lemma 3.3. Suppose that $M$ is a pwl manifold, $Y \subseteq M, \mu \in C(M, Y)$, and $k$ is a natural number.

There is a function $\mu_{1} \in C(M, Y)$ sucb that if $Q$ and $P$ are polybedra in $Y$ with $Q=Q_{0} \searrow Q_{1} \searrow \cdots \searrow Q_{k}=P$ where each $Q_{i-1} \searrow Q_{i}$ is a $\mu_{1}$-collapse admissible with respect to $\mathrm{Bd} M$ and if $N$ is a $\mu_{1}$-regular neigbborbood of $Q$ in $M$, then $N$ is a $\mu$-regular neighborbood of $P$ in $M$.

Proof. Use Lemma 2.1 to find $\mu_{2}, \cdots, \mu_{5}, \mu_{1} \in C(M, Y)$ so that $\left(M, \mu / 6, \mu_{2}, 2\right)$, $\left(M, \mu_{2} / 2 k, \mu_{3}, 2 k+1\right),\left(M, \mu_{3} / 2, \mu_{4}, 2\right),\left(M, \mu_{4} / 2,2 \mu_{5}, 3\right)$, and $\left(M, \mu_{5}, \mu_{1}, 2\right)$ all 
have Property S. Then choose a triangulation $(J,\{J\}, \phi)$ of $\left(M,\left\{Q_{i}\right\}\right)$ so that, for each simplex $t$ of $J, \phi(t) \cap Q \neq \varnothing$ implies that $\phi(N(t, J))$ is a $\mu_{1}$-set. From the proofs of Theorems 8.1 and 9.1 of [7] and Theorem 2 of [23] (each time a triangulation is mentioned make sure that the triangulation induces a subdivision of $J$ ) there correspond to each collapse $Q_{i-1} \searrow Q_{i}$ a pair of $\mu_{1}$-regular neighborhoods $N_{i-1}$ of $Q_{i-1}$ and $N_{i}^{\prime}$ of $Q_{i}$ and a pwl $\mu_{1}$-homeomorphism $b_{2 i}$ of $M$ onto itself which is the identity on $Q_{i}$ and takes $N_{i-1}$ onto $N_{i}^{\prime}$. From Lemma 3.1 there is a pwl $\mu_{4}$-homeomorphism $b_{1}$ of $M$ onto itself which is the identity on $Q$ and takes $N$ onto $N_{0}$ and for each $i<k$ there is a pwl $\mu_{4}$-homeomorphism $b_{2 i+1}$ of $M$ onto itself which is the identity on $Q_{i}$ and takes $N_{i}^{\prime}$ onto $N_{i}$. Set $b=b_{2 k} b_{2 k-1} \cdots b_{1}$. Each $b_{i}^{-1}$ is a $\mu_{3}$-homeomorphism so $b^{-1}$ is a $\mu_{2}$-homeomorphism. It follows from Lemma 3.2 that $N=b^{-1}\left(N_{k}^{\prime}\right)$ is a $\mu$-regular neighborhood of $P$.

Lemma 3.4. Suppose $M$ is a pwl n-manifold, $Q$ is a pwl sub-n-manifold which is closed in $M$ and has nonempty frontier, $Q \cap \mathrm{Bd} M$ is either a pwl $(n-1)$-manifold in $\mathrm{Bd} Q$ or the empty set, and $\mu \in C(M)$.

If $\lambda:(\operatorname{Fr} Q) \times[0, r] \rightarrow \mathrm{Cl}(M-Q)$ is a proper, pwl $\mu$-collar on $\operatorname{Fr} Q$ in $\mathrm{Cl}(M-Q)$, then $Q \cup$ image $\lambda$ is a $\mu$-regular neighborbood of $Q$.

Proof. It follows from the Newman theorem (see [27, Theorem 3] for example) that $\mathrm{Cl}(M-(Q \cup$ image $\lambda))$ is a pwl $n$-manifold. Use Lemma 2.4 to extend $\lambda$ to $\lambda^{\prime}$ so that $\lambda^{\prime}$ takes $(\operatorname{Fr} Q) \times[0, r+1]$ into $\mathrm{Cl}(M-Q)$ as a proper, pwl $\mu$-collar. Let $(\tau, \phi)$ be a triangulation of $\operatorname{Fr} Q$ such that $\lambda^{\prime}(\phi(N(t, \tau)) \times[0, r+11)$ is a $\mu$-set for each $t \in \tau$. Let $T$ be a subdivision of the cell complex $\tau \times[0, \tau+1]$ which adds no vertices (see [27, Lemma 1]), and let $\psi:|T| \rightarrow M$ be given by

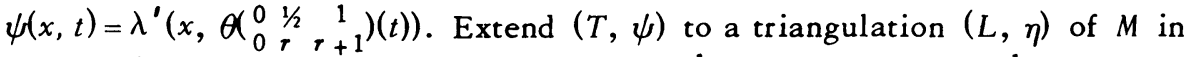
which $\eta(N(t, L))$ is a $\mu$-set for each $t \in\left[\eta^{-1}(Q)\right]$. Note that $\left[\eta^{-1}(Q)\right]$ is automatically a full subcomplex $K$ of $L$. Then $\eta\left(N\left(K^{1}, L^{1}\right)\right)=Q \cup$ image $\lambda$ where $L^{1}$ is the first barycentric subdivision of $L$. Thus $Q \cup$ image $\lambda$ is a $\mu$-regular neighborhood of $Q$.

Corollary 3.5. Suppose $M$ is a pwl n-manifold, $Y \subseteq M, \mu \in C(M, Y)$, and $k$ is a natural number.

There is a function $\mu_{1} \in C(M, Y)$ such that if $P$ and $N$ are polybedra in $Y$ where $N$ is a pwl n-manifold containing a neighborbood of $P, N \cap \mathrm{Bd} M$ is either a pwl $(n-1)$-manifold or the empty set, and $\left.N=N_{0} \searrow \cdots\right\rangle N_{k}=P$ is a sequence of $k \mu_{1}$-collapses admissible with respect to $\mathrm{Bd} M$, then $N$ is a $\mu$-regular neighborbood of $P$.

Proof. Choose $\mu_{1}$ from Lemma 3.3 corresponding to $M, Y, \mu$, and $k$. Let $N=N_{0} \searrow \ldots \searrow \quad N_{k}=P$ be given as in the hypothesis. Choose $\mu_{2} \in C(M, Y)$ so small that $\mu_{2} \leq \mu_{1}$ and so that if $b$ is a $\mu_{2}$-homeomorphism of $M$ onto itself then 
each of the collapses $b\left(N_{i-1}\right) \searrow b\left(N_{i}\right)$ is still a $\mu_{1}$-collapse. The Newman theorem cited in the preceding proof shows that $\mathrm{Cl}(M-N)$ is a pwl $n$-manifold and that $\operatorname{Fr} N$ is a pwl $(n-1)$-manifold. Use Lemma 2.4 to find a pwl embedding $\lambda:(\operatorname{Fr} N) \times[-1,2] \rightarrow M-P$ so that each $\lambda(y \times[-1,2])$ is a $\mu_{2}$-set, $\lambda \mid(\operatorname{Fr} N) \times$ $[-1,0]$ is a proper collar on $\operatorname{Fr} N$ in $\mathrm{Cl}(M-N)$, and $\lambda \mid(\operatorname{Fr} N) \times[0,2]$ is a proper collar on $\operatorname{Fr} N$ in $N$.

Define a pwl $\mu_{2}$-homeomorphism $b$ of $M$ onto itself by $b=I$ on $M$-image $\lambda$ and $b \lambda(y, t)=\lambda\left(y, \theta\left(\begin{array}{lll}-1 & 0 & 2 \\ -1 & 1 & 2\end{array}\right)(t)\right)$. Set $Q=b(N)$. By Lemma 3.4, $N$ is a $\mu_{2}$-regular neighborhood of $Q$, and by Lemma 3.3 and the conditions on $\mu_{2}, N$ is a $\mu$-regular neighborhood of $P$.

Lemma 3.6. Suppose $M$ is a pwl n-manifold, $Q$ is a polybedron in $M$, and $\mu \epsilon$ $C(M, Q)$.

Then there are $\mu$-regular neighborboods of $Q$, and if $N$ is any $\mu$-regular neighborbood of $Q$, then $N=N_{0} \searrow \cdots \searrow N_{n}=Q$ by a sequence of $n \mu$-collapses each of which is admissible with respect to $\mathrm{Bd} M$.

Proof. The existence of $\mu$-regular neighborhoods follows from [7] or [231 and the fact there are triangulations of neighborhoods of $Q$ in $M$ which partition the neighborhoods into appropriately small sets.

Let $N$ be a $\mu$-regular neighborhood of $Q$ in $M$ with $N=\phi\left(N\left(K^{1}, J^{1}\right)\right)$ where $(J, K, \phi)$ is a triangulation of $(M, Q)$ with $K \triangleleft J, J^{1}$ is a first derived subdivision of $J$, and for each $t \in K, \phi(N(t, J))$ is a $\mu$-set. Define another first derived subdivision $J^{\prime}$ of $J$ as in [27, Chapter 3$]$ by first defining a simplicial map $\lambda: J \rightarrow[0,1]$ with $\lambda(v)=0$ if $v \in K_{0}$ and $\lambda(v)=1$ if $v \in J_{0}-K_{0}$. Choose $J^{\prime}$ so that if $\sigma$ is a simplex of $J$ with $\lambda(\sigma)=[0,1]$ then $d(\sigma) \in \lambda^{-1}(1 / 2)$.

Let $J^{\prime \prime}$ be the first barycentric subdivision of $J^{\prime}$ and $K^{\prime \prime}$ the induced subdivision of $K$. Let $\left\{t_{i}^{j}\right\}$ denote the $j$-simplexes of $K$. Set $D_{j}=\bigcup_{i} N\left(d\left(t_{i}^{j}\right), K^{\prime \prime}\right)$, and for each simplex $t$ of $K$ let $t(j)=D_{j} \cap t$. Set $N_{0}^{\prime}=N\left(K^{\prime}, J^{\prime}\right)$ and $N_{k}^{\prime}=\phi^{-1}(Q) \cup$ $\left(N_{0}^{\prime} \cap(\bigcup\{t(j) * s: t \in K, \lambda(s)=1, t * s \in J\right.$, and $\left.j<n-k\})\right)$. We claim that each $N_{k-1}^{\prime}$ collapses to $N_{k}^{\prime}$ by a collapse that is admissible with respect to $\phi^{-1}(\mathrm{Bd} M)$. To see this look at a simplex $s * t$ of $J$ with $\lambda(s)=1$ and $\lambda(t)=0$. Then for $j=$ $n-k, C=(t(j) * s) \cap N_{0}^{\prime} \subseteq N_{k-1}^{\prime}$ is a pwl cell. Furthermore, $C \cap N_{k}^{\prime}$ is a collapsible polyhedron in $\mathrm{Bd} C$, so $C \searrow C \cap N_{k}^{\prime}$. An induction argument now shows that $N_{k-1}^{\prime} \searrow N_{k}^{\prime}$ and that this collapse is admissible with respect to $\phi^{-1}(\mathrm{Bd} M)$.

Let $\eta: f^{\prime} \rightarrow J^{1}$ be a simplicial is omorphism which is the identity on $J_{0}$. Set $N_{k}=\phi \eta\left(N_{k}^{\prime}\right)(k=0, \cdots, n)$. Each $N_{k-1} \searrow N_{k}$ is a $\mu$-collapse which is admissible with respect to $\mathrm{Bd} M$, and $N_{n}=Q$.

4. Extending the Lininger-Hosay theorem. Here we use a result of Lister's [181 to generalize the theorem of Lininger and Hosay's [15], [17] on re-embedding crumpled cubes in $E^{3}$. The trick of using [18] to simplify the proof of the 
re-embedding theorem was observed by Daverman in [12].

Lemma 4.1. Suppose $S$ is a 2-sphere in $E^{3}, D$ is a disk on $S$ such that $\mathrm{Cl}(S-D)$ is tame, and $U$ is a connected open set in $E^{3}$ sucb that $U \cap S=$ Int $D$ and $U-S$ has two components $-U_{0}$ and $U_{1}$.

There is a bomeomorphism $g$ of $S$ onto a tame 2-sphere in $E^{3}$ and there is a bomeomorphism $b$ of $E^{3}-U_{1}$ into $E^{3}$ such that

(1) $b \mid E^{3}-U=I$,

(2) $g \mid S-$ Int $D=I$,

(3) $g($ Int $D) \subseteq U$, and

(4) $b\left(E^{3}-U_{1}\right) \cap g($ Int $D)=\varnothing$.

Proof. There is no loss in supposing that $U_{0} \subseteq$ Ext $S$ and $\operatorname{Fr} U$ is connected. Since $E^{3}$ is unicoherent the second condition can be achieved by cutting down $U$ to a smaller open set if necessary. Define $\mu \in C(S)$ by $\mu(x)=d\left(x, E^{3}-U\right) / 2$. From [18, Theorem 5] there is a homeomorphism $f$ of $S$ into $E^{3}$ and there is a null sequence $E_{1}, \cdots, E_{i}, \cdots$ of mutually exclusive disks in Int $D$ so that (1) $\bigcup_{E_{i}}$ is closed in Int $D$, (2) $f \mu$-approximates the identity, (3) $f($ Int $D)$ is locally

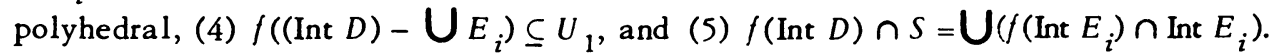
By [3, Theorem 8.5], $f(S)$ is tame, so $B=\mathrm{Cl}$ Int $f(S)$ is a 3-cell.

For each $i$, set $F_{i}=f\left(E_{i}\right)$. We can suppose that each $F_{i}$ is a polyhedron. Let $G_{i}$ denote the sum of all components of $B \cap \mathrm{Cl}$ Ext $S$ which intersect $F_{i}$. Because $\operatorname{Fr} G_{i} \subseteq U$ and $\operatorname{Fr} U$ is connected, $G_{i} \subseteq \mathrm{Cl}_{0}$. It follows as in [12] that the $G_{i}$ 's are mutually exclusive.

For each $F_{i}$ let $B_{i}$ be a 3 -cell in $F_{i} \cup$ Int $B$ such that $B_{i} \cap f(S)=F_{i}, G_{i} \subseteq$ $B_{i}$, and $G_{i} \cap \operatorname{Bd} B_{i} \subseteq F_{i}$. Let $C_{1}, \cdots, C_{i}, \cdots$ be a null sequence of mutually exclusive polyhedral cubes in $B \cap U$ so that each $C_{i} \cap f(S)=F_{i}$. For each $B_{i}$ let $\phi_{i}$ be a homeomorphism of $B_{i}$ onto $C_{i}$ which is the identity on $F_{i}$.

Define $g$ to be $f$ on $S-\bigcup E_{i}$ and let $g$ take each $E_{i}$ onto $\left(\operatorname{Bd} C_{i}\right)-\operatorname{Int} F_{i}$. Set $b(x)=x$ for $x \in \mathrm{Cl}$ Ext $f(S)$ and $b(x)=\phi_{i}(x)$ for $x \in G_{i}$. Just as in [12], $g$ and $b$ satisfy conditions (1)-(4).

Lemma 4.2. Suppose $M$ is a connected 3-manifold without boundary, $S$ is a connected boundaryless surface in $M$ which is a closed subset of $M$, and $S$ sep. arates $M$ into two components $-V_{0}$ and $V_{1}$.

Suppose $\mu \in C(M, S)$.

Then there is a $\mu$-bomeomorphism $g$ of $S$ onto a tame surface in $M$ and there is a $\mu$-bomeomorphism $b$ of $\mathrm{Cl} V_{0}$ into $M$ so that $g(S) \cap b\left(\mathrm{Cl} V_{0}\right)=\emptyset$.

Proof. First cover $S$ with a collection of open 3-cells in $M$. Then use [5, Theorem 6.3] and [4, Theorem 5] to find a curvilinear triangulation $T$ of $S$ with tame 1-skeleton $T_{1}$ and 2-simplexes $\left\{\Delta_{i}\right\}$ so that each $\Delta_{i} \subseteq S_{i} \subseteq N_{i}$ where $S_{i}$ is 
a 2-sphere and $N_{i}$ is an open 3-cell which is a $\mu / 2$-set. From [5] we can assume that each $S_{i}$ is locally tame modulo $\Delta_{i}$. Use [8, Lemma 2.1] to find for each $\Delta_{i}$ a connected open set $U_{i}$ in $N_{i}$ such that Int $\Delta_{i} \subseteq U_{i}, S S_{i} \cap \mathrm{Cl} U_{i}=\Delta_{i}$, and $U_{i}-$ $\Delta_{i}$ has exactly two components. Choose the $U_{i}$ 's so that they are mutually exclusive. For each $U_{i}$ set $U_{i 0}=U_{i} \cap V_{0}$ and $U_{i 1}=U_{i} \cap V_{1}$.

Use Lemma 4.1 to find for each $\Delta_{i}$ a homeomorphism $f_{i}$ of $S_{i}$ into $N_{i}$ and a homeomorphism $b_{i}$ of $N_{i}-U_{i 1}$ into $N_{i}$ such that $f_{i}=I$ on $S-\operatorname{Int} \Delta_{i}, f_{i}\left(\right.$ Int $\left.\Delta_{i}\right)$ is locally tame, $b_{i}=I$ on $N_{i}-U_{i}$, and $b_{i}\left(N_{i}-U_{i 1}\right)$ fails to intersect $f_{i}\left(\right.$ Int $\left.\Delta_{i}\right)$. Set $b=I$ on $M-\bigcup U_{i}$ and $b=b_{i}$ on each $\mathrm{Cl} U_{i 0}$. Set $f=f_{i}$ on each $\Delta_{i}$. From [5, Theorem 3.1] and [1], [22], $f(S)$ is tame. The condition on the diameters of the $N_{i}$ 's shows that $b$ is a $\mu$-homeomorphism and that $f$ is a $\mu / 2$-approximation to the identity.

Since each $f\left(\Delta_{i}\right)$ separates $U_{i}$ it follows that $f(S)$ separates $M$ into components $W_{0}$ and $W_{1}$ where $W_{0}$ is the component which contains the connected set $h\left(M-\left(V_{1} \cup T_{1}\right)\right)$. By Lemma 2.4 we can push $f(S)$ off into $W_{1}$ to get a $\mu$-homeomorphism $g$ of $S$ onto a tame surface in $W_{1}$. This completes the proof of the theorem.

We can relax some what the requirement that $S$ have no boundary:

Corollary 4.3. Suppose $M$ is a connected 3-manifold, $S$ is a connected surface in $M$ with $S \cap \mathrm{Bd} M=\mathrm{Bd} S$ and $S$ is closed in $M$, and suppose $S$ separates $M$ into two components $-V_{0}$ and $V_{1}$.

Suppose $\mu \in C(M, S)$.

Then there is a $\mu$-bomeomorphism $g$ of $(S, \mathrm{Bd} S)$ onto a tame pair in $(M, \mathrm{Bd} M)$ and there is a $\mu$-bomeomorphism $b$ of $\left(\mathrm{Cl} V_{0}\right.$, ( $\left.\left.\mathrm{Bd} M\right) \cap \mathrm{Cl} V_{0}\right)$ into $(M, \mathrm{Bd} M)$ so that $\mathrm{g}(S) \cap b\left(\mathrm{Cl} V_{0}\right)=\varnothing$.

Proof. Let $S^{\prime} \subseteq M^{\prime}$ be the doubles of $S$ and $M$ along $\mathrm{Bd} S$ and $\mathrm{Bd} M$. Here $M^{\prime}$ is obtained by attaching two copies of $M$ together with the identity homeomorphism on $\mathrm{Bd} M$. Extend $\mu$ to $\mu^{\prime} \in C\left(M^{\prime}, S^{\prime}\right)$. Carry out the constructions in the proof of Lemma 4.2 for $S^{\prime}$ in $M^{\prime}$ to get $f^{\prime}, g^{\prime}$, and $b^{\prime}$ in place of $f, g$, and $b$ with the following modifications: (1) Require that $\mathrm{Bd} S$ be contained in $T_{1}$, the tame 1-skeleton of the curvilinear triangulation of $S^{\prime} ;(2)$ choose the $U_{i}$ 's so that each $U_{i} \cap \mathrm{Bd} M=\emptyset$; and (3) in defining $g^{\prime}$ from $f^{\prime}$ by pushing off $T_{1}$ require that $\left(g^{\prime}\right)^{-1}(\operatorname{Bd} M)=\operatorname{Bd} S$. Then for $b$ and $g$ just take $b^{\prime} \mid M$ and $g^{\prime} \mid S$.

The theorem which follows, the promised extension of the Lininger-Hosay theorem, is obtained from Lemma 4.3 in much the same way as Theorem 2 is established in [17]. We give only a sketch of the proof.

Theorem 4.4. Suppose $M$ is a connected 3-manifold, $S$ is a connected surface in $M$ with $S \cap \mathrm{Bd} M=\mathrm{Bd} S$ and $S$ is a closed subset of $M$, and suppose $S$ separates $M$ into two components $-V_{0}$ and $V_{1}$. 
Suppose $\mu \in C(M, S)$.

Then there is a $\mu$-homeomorphism $b$ of $\left(M-V_{1},(\mathrm{Bd} M)-V_{1}\right)$ into $(M, \mathrm{Bd} M)$ such that $b(S)$ is collared in $M-b\left(V_{0}\right)$.

Proof. By combining Corollary 4.3 with [9, Theorem 9.2] it is possible to define inductively a sequence of re-embeddings $b_{i}(i=1,2, \ldots)$ of $\left(\mathrm{Cl} V_{0}\right.$, $\left.(\mathrm{Bd} M) \cap \mathrm{Cl} V_{0}\right)$ into $(M, \mathrm{Bd} M)$, a sequence of homeomorphisms $g_{i}(i=1,2, \cdots)$ of $(S, S \cap \mathrm{Bd} M)$ onto tame pairs in $(M, \mathrm{Bd} M)$, and an embedding $\lambda$ of $(S \times[0,1]$, $(S \cap \operatorname{Bd} M) \times[0,1])$ into $(M, B d M)$ so that (1) $b_{i} \rightarrow b$ a $\mu$-homeomorphism of $\mathrm{Cl} V_{0}$ into $M$, (2) $g_{i} \rightarrow b \mid S$ and $g_{i}(S) \cap b\left(\mathrm{Cl} V_{0}\right)=\emptyset$ for each $i$, and (3) $\lambda(y, 1 / i)=g_{i}(y)(y \in S, i=1,2, \cdots)$. But this says that $h(S)$ is collared in $M-b\left(V_{0}\right)$.

5. Some combinatorial preliminaries. We omit a proof of the first lemma here. It is easily obtained by building up a homeomorphism over blocks of convex linear cells.

Lemma 5.1. Suppose $u * v * S=u * B$ is a combinatorial $n$-ball where $u$ and $v$ are vertices and $S$ is a combinatorial $(n-2)$-sphere. Let $r$ denote the simplicial retraction $u * B \rightarrow B$ which sends $u$ to $v$.

Then there is a pwl homeomorphism $\phi$ of $|u * B|-|S|$ onto $\mid$ Int $B \mid \times[0,1]$ sucb that, for eacb $y \in$ Int $B, \phi r^{-1}(y)=y \times[0,1]$ and $\phi(y)=(y, 0)$.

Lemma 5.2. Suppose $K_{a} \triangleleft K$ and $L_{a} \triangleleft L$ are complexes, $r$ is a simplicial map from $L$ to $K, v$ is a vertex of $K$, and $S$ is a 1 -sphere in $1 \mathrm{k}(v, K)$ such that $\left(L, L_{a}, r\right)$ is an elementary reduction of $\left(K, K_{a}\right)$ at $(\nu, S)$.

If $H$ and $J$ are finite subcomplexes of $K$ containing $v$ sucb that $H \gamma^{s} J$ by a collapse that is admissible with respect to $K_{a^{\prime}}$ then $r^{-1}(H) \searrow^{s} r^{-1}(J)$ by a collapse that is admis sible with respect to $L_{a}$. Furthermore, $r^{-1}(v) \searrow^{s} 0$ by a collapse that is admissible with respect to $L_{a}$.

Proof. First collapse $r^{-1}(H) \searrow^{s} H(0) \cup r^{-1}(J)$ where $H(0)=\left[\left|r^{-1}(H)\right|-\right.$ $(|v * u * S|-|v * S|)]$ and $u$ is the new vertex added to obtain $L$. Do this by collapsing simplexes $v * u * r^{-1}(t)$ across free faces $u * r^{-1}(t)$ in order of decreasing dimension for simplexes $t$ of $S \cap(H-J)$. Then for each simplex $t \in H$ with $t \neq v$, there is a unique simplex $\tilde{t}$ in $H(0)$ which is taken isomorphically onto $t$ so $H \searrow^{s} J$ induces a collapse $H(0) \cup r^{-1}(J) \searrow^{s} r^{-1}(J)$ via $r^{-1}$. It is clear that this collapse is admissible with respect to $L_{a}$ and that $r^{-1}(v) \searrow^{s} 0$ by a collapse that is admissible with respect to $L_{a}$.

6. Splitting a surface. Theorem 6.1 is the key to reducing the canonical neighborhood problem to one that can be solved using McMillan's techniques. It says roughly that a two sided surface $S$ in a 3 -manifold can be split open and 
blown up into $S \times[0,1]$. McMillan uses this idea in [21] in the proof of Theorem 2 when he constructs the auxiliary manifold $M^{*}$. Daverman and Eaton [13] have sketched out a weaker version of this theorem for interiors of disks.

Theorem 6.1. Suppose $P_{a}<P$ and $Q_{a}<Q$ are polybedra, $r$ is a pwl map from $\left(Q, Q_{a}\right)$ to $\left(P, P_{a}\right), S$ is a connected polybedral surface in $P$ with $S \cap P_{a}=$ $\mathrm{Bd} S$, and $\phi$ is a pwl homeomorphism from $(S \times[0,1],(\mathrm{Bd} S) \times[0,1])$ to a polybedral pair $\left(C, C_{a}\right)<\left(Q, Q_{a}\right)$ so that, on $C, r \phi$ is the projection of $S \times[0,1]$ onto $S$. Suppose that $r$ takes $Q-C$ bomeomorphically onto $P-S$.

Suppose $M$ is a 3-manifold and $f:\left(P, P_{a}\right) \rightarrow(M, \mathrm{Bd} M)$ is a closed embedding, and suppose there is a connected open set $W$ in $M$ containing $f(S)$ such that $W-S$ has two components $-W_{0}$ and $W_{1}$ with $\mathrm{Cl}\left(r^{-1} f^{-1}\left(W_{i}\right)\right) \cap C \subseteq S_{i}=$ $\phi(S \times i)(i=0,1)$.

Suppose $\mu \in C(M, f(S))$.

Then there is an embedding $g:\left(Q, Q_{a}\right) \rightarrow(M, \mathrm{Bd} M)$ and there is a special map $\pi$ for $(M, f, g, r, \mu)$.

Proof. The idea of the proof is to use Theorem 4.4 to obtain from $f$ embeddings of $f^{-1}\left(\mathrm{Cl}_{i}\right) \quad(i=0,1)$ whose boundaries are collared on one side and then to use [10, Theorem 8.2] to separate the two copies of $f(S)$ so that the collars attach along corresponding endpoints. See Figure 6.1.
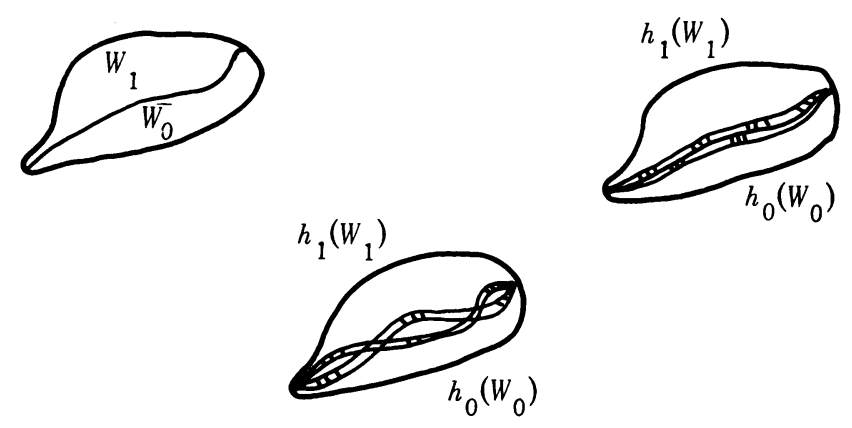

Figure 6.1

Let $U$ be a connected open set in $M$ such that $S \subseteq U \subseteq W$ and $X_{i}=(\operatorname{Fr} U) \cap$ $W_{i} \neq \emptyset(i=0,1)$. Set $U_{i}=U \cap W_{i}(i=0,1)$. Let $\mu_{1}$ and $\mu_{2} \in C(M, f(S), M-U)$ be such that $\left(M, \mu_{1}, 2 \mu_{2}, 2\right)$ and $\left(M, \mu_{1}, \mu_{2}, 2\right)$ have Property $\mathrm{S}$. Substitute $\left(U \rightarrow M, S \rightarrow K, \mathrm{Bd} S \rightarrow K_{a}, f \rightarrow f, \mu_{2} \rightarrow \mu\right)$ in Theorem 8.2 of [10] to find an associated $\nu \in C(S, S)$. Use Theorem 4.4 to find $\mu_{2}$-homeomorphisms $b_{0}$ of $M-U_{1}$ and $b_{1}$ of $M-U_{0}$ so that $b_{i} f(S)$ is locally collared in $M-b_{i}\left(U_{i}\right)$ and $\rho\left(f(x), b_{i} f(x)\right)<\nu(x)(x \in S, i=0,1)$. Set $S_{1 / 2}=\phi(S \times 1 / 2)$, and set $C_{i}=$ $\phi(S \times[i, 1 / 2])(i=0,1)$. (Here $[1,1 / 2]=[1 / 2,1]$.) From Lemma 2.4 there are homeomorphisms $g_{i}$ of $C_{i}$ into $U-b_{i}\left(U_{i}\right)(i=0,1)$ so that $(1) g_{i}\left(C_{i}-S_{i}\right)$ is locally 
tame, (2) for each $x \in S_{i}, g_{i}(x)=h_{i} f r(x)$, (3) for each $x \in S, g_{i}\left(r^{-1}(x) \cap C_{i}\right)$ is a $\mu_{2} f(x)$-set, and (4) for each $x \in C_{i}, \rho\left(g_{i}(x), f r(x)\right)<\nu r(x) \leq \mu_{2}(f r(x))$.

Define homeomorphisms $f_{i}$ of $S$ into $U(i=0,1)$ by $f_{i}(x)=g_{i}\left(r^{-1}(x) \cap S_{1 / 2}\right)$. For each $x \in S, \rho\left(f(x), f_{i}(x)\right)<\nu(x)$ by (4) in the preceding paragraph. Now $f_{i}(S)$ is tame in $U$ by [1], [22] so by our application of [10] there is a $\mu_{2}$-homeomorphism $b$ of $M$ such that $b f_{0}=f_{1}$.

Note that $f_{1}(S)$ separates $X_{0}$ from $X_{1}$ in $\mathrm{Cl} U$ because $b_{1}\left(\mathrm{Cl} U_{1}\right) \cup$ $g_{1}\left(C_{1}-S_{1 / 2}\right)$ is a sum of components of $(\mathrm{Cl} U)-f_{1}(S)$ which contains $X_{1}$ and misses $X_{0}$. Furthermore, $b_{1}\left(\mathrm{Cl} U_{1}\right) \cap f_{1}(S)=b b_{0}\left(\mathrm{Cl} U_{0}\right) \cap f_{1}(S)=\varnothing$. Thus $b_{1}\left(\mathrm{Cl} U_{1}\right) \cap b b_{0}\left(\mathrm{Cl} U_{0}\right)=\varnothing$, and $g$ is a homeomorphism where $g$ is defined by $g=f r$ on $Q-r^{-1} f^{-1}(U), g=b b_{0} f$ on $r^{-1} f^{-1}\left(U_{0}\right), g=b_{1} f$ on $r^{-1} f^{-1}\left(U_{1}\right), g=$ $b g_{0}$ on $C_{0}$, and $g=g_{1}$ on $C_{1}$. Notice that $U-g(C)=b b_{0}\left(U_{0}\right) \cup b_{1}\left(U_{1}\right)$.

Define $\pi$ by $\pi=I$ on $M-U, \pi=b_{0}^{-1} b^{-1}$ on $b b_{0}\left(\mathrm{Cl} U_{0}\right), \pi^{\cdot}=b_{1}^{-1}$ on $b_{1}\left(\mathrm{Cl} U_{1}\right)$, and $\pi=\operatorname{frg}^{-1}$ on $g(C)$.

If $x \in b_{1}\left(\mathrm{Cl}_{1}\right)$ with $x=b_{1}(y)$ then $\rho(x, \pi(x))=\rho(x, y) \leq \mu_{2}(y) \leq \mu(x)$. If $x \in b b_{0}\left(\mathrm{Cl} U_{0}\right)$ then $x=b b_{0}(y)$ and $\pi(x)=y$. Now $\rho\left(y, b_{0}(y)\right) \leq \mu_{2}(y) \leq \mu_{1}\left(b_{0}(y)\right)$ $\leq \mu(x) / 2$, and $\rho\left(b_{0}(y), x\right) \leq \mu_{2}\left(b_{0}(y)\right) \leq \mu_{1}(x) \leq \mu(x) / 2$. Thus $\rho(x, \pi(x)) \leq \mu(x)$. If $x \in g(C)$, then $x=g(y)$ and $\pi(x)=f r(y)$. By an analysis similar to the preceding one we find that $\rho(x, \pi(x)) \leq \mu(x)$ for $x \in g(C)$.

It remains to show that $\pi$ can be approximated arbitrarily closely by a homeomorphism. Since point inverses $\pi^{-1}(x)$ are cellular [19, Theorem 6] this actually follows from work of Voxman's [25, Theorem 1] (Bd $M$ presents no real problem); however it is instructive to look at a direct proof.

Let $\lambda \in C\left(M, \mu^{-1}(0, \infty)\right)$. Choose $\lambda_{1}$ and $\lambda_{2} \in C\left(M, \mu^{-1}(0, \infty)\right)$ so that, for $x \in M, \lambda_{1}(x) \leq \inf \lambda \mid \pi^{-1}(x)$ and so that $\left(M, \lambda_{1}, 2 \lambda_{2}, 2\right)$ has Property S. Repeat the construction of $g$ and $\pi$ with $\lambda_{2}$ in place of $\mu$ to get $\lambda_{2}$-homeomorphisms $b_{0}^{\prime}$ of $M-U_{1}, b_{1}^{\prime}$ of $M-U_{0}, b^{\prime}$ of $M$, a homeomorphism $g^{\prime}$ of $Q$ and a $\lambda_{2}$-map $\pi^{\prime}$ such that $\pi^{\prime} g^{\prime}=f r$. Define an approximation $H$ to $\pi$ by $H=I$ on $M-U, H=$ $h_{1}^{\prime} b_{1}^{-1}$ on $b_{1}\left(\mathrm{Cl} U_{1}\right), H=b^{\prime} b_{0}^{\prime}\left(b b_{0}\right)^{-1}$ on $b b_{0}\left(\mathrm{Cl} U_{0}\right)$, and $H=g^{\prime} g^{-1}$ on $g(C)$. Clearly, $H$ is a homeomorphism. If $x \in b_{1}\left(\mathrm{Cl} U_{1}\right)$, then $x=b_{1}(y)$ and $H(x)=$ $b_{1}^{\prime}(y)=b_{1}^{\prime} \pi(x)$. Thus $\rho(H(x), \pi(x)) \leq \lambda_{2}(y) \leq \lambda_{1}(y) \leq \lambda(x)$ by the definition of $\lambda_{1}$. If $x \in b b_{0}\left(\mathrm{Cl} U_{1}\right)$, then $x=b h_{0}(y)$, and $H(x)=b^{\prime} b_{0}^{\prime}(y)=b^{\prime} b_{0}^{\prime} \pi(x)$. Now $\rho\left(b^{\prime} h_{0}^{\prime} \pi(x)_{2} \pi(x)\right)$ $\leq \lambda_{1}(x)$ since $b^{\prime} b_{0}^{\prime}$ is a $\lambda_{1}$-homeomorphism. Thus $\rho\left(b^{\prime} b_{0}^{\prime} b_{0}^{-1} b^{-1}(x), \pi(x)\right) \leq \lambda(x)$. For $x \in g(C)$ we have $x=g(y)$ and $\pi(x)=g r(y)$. Now $\rho(H(x), \pi(x))=$ $\rho\left(g^{\prime}(y), \pi^{\prime} g^{\prime}(y)\right) \leq \lambda_{2}\left(g^{\prime}(y)\right) \leq \lambda_{1}\left(\pi^{\prime} g^{\prime}(y)\right)=\lambda_{1}(\pi(x)) \leq \lambda(x)$.

For our work here we need to allow $f^{-1}(\mathrm{Bd} M)$ to contain points in Int $S$. The corollary which follows enables us to do this:

Corollary 6.2. Suppose $P_{a}<P$ and $Q_{a}<Q$ are polybedra, $r$ is a pwl map from $Q$ to $P$ which takes $Q_{a}$ onto $P_{a}, S$ is a connected polybedral surface in $P$ 
with $\mathrm{Bd} S=\emptyset$, and $\phi: S \times[0,1] \rightarrow C \subseteq Q$ is a pwl bomeomorphism such that $r \phi$ is the projection of $S \times[0,1]$ on $S$. Suppose $r$ takes $Q-C$ bomeomorphically onto $P-S$. Set $S_{i}=\phi(S \times i)(i=0,1)$. Suppose $Q_{a} \cap C=S_{1} \cap r^{-1}\left(P_{a}\right)$.

Suppose $M$ is a pwl 3-manifold, $f:\left(P, P_{a}\right) \rightarrow(M, \mathrm{Bd} M)$ is a closed embedding, and suppose $\mu \in C(M, f(S))$.

Let $M^{\prime}$ denote the sum of $M$ and $(\mathrm{Bd} M) \times[0,1]$ with the identification $y=$ $(y, 0)$ on $\mathrm{Bd} M$. Suppose that there is a connected open set $W$ in $M^{\prime}$ containing $f(S)$ which is separated by $f(S)$ into two components $-W_{0}$ and $W_{1}$ with $W_{0} \subseteq$ Int $M$ and $\left(\mathrm{Cl} r^{-1} f^{-1}\left(W_{i}\right)\right) \cap C \subseteq S_{i}(i=0,1)$. Suppose that for each $x \in P_{a} \cap \mathrm{Cl}\left(S-P_{a}\right)$ there is a subpolybedron $P(x)$ in $f^{-1}\left(\mathrm{Cl} W_{1}\right)$ sucb that $P(x)$ contains a neighborbood of $x$ in $S, P(x)$ bas no local cut points, $P_{a}(x)=P(x) \cap P_{a}$ bas no point components, and $P(x)-P_{a}(x)$ is a 3-manifold.

Then there is a closed embedding $g:\left(Q, Q_{a}\right) \rightarrow(M, \mathrm{Bd} M)$ and there is a special map $\pi$ for $(M, f, g, r, \mu)$ which is the identity on $\mathrm{Bd} M$.

Proof. Extend the metric on $M$ to one on $M^{\prime}$, and extend $\mu$ to a function in $C\left(M^{\prime}\right)$. Replace $M$ by $M^{\prime}$ in the proof of Theorem 6.1 and carry out the constructions there. Since $\pi \mid b_{1}\left(M^{\prime}-\right.$ Int $\left.M\right)=b_{1}^{-1} \ldots$, it is sufficient to show that $b_{1}$ can be chosen so that it is the identity on $M^{\prime}-$ Int $M$.

Following the notation in the proof of Theorem 6.1, define a $\mu_{2}$-homeomorphism $b_{3}$ of $M^{\prime}-U_{0}$ such that $b_{3} \mid M^{\prime}-M=I, b_{3} f\left(S-P_{a}\right)$ is locally collared in $M^{\prime}-b_{3}\left(U_{1}\right)$, and $\rho\left(f(x), b_{3} f(x)\right)<\nu(x)(x \in S)$. We claim that $b_{3} f(S)$ is locally collared in $M-b_{3}\left(U_{1}\right)$. The only points where this could fail to be so are points in $f\left(P_{a} \cap \mathrm{Cl}\left(S-P_{a}\right)\right)$. Let $x \in f\left(P_{a} \cap \mathrm{Cl}\left(S-P_{a}\right)\right)$. Then from [10, Lemma 10.1], $b_{3}(P(x))$ is locally tame at $x$ and so $b_{3} f(S)$ is locally collared at $x$ in $M-b_{3}\left(U_{1}\right)$.

This shows that we could have used $b_{3}$ in place of $b_{1}$ and the proof of the corollary is complete.

Theorem 6.3. Suppose $K$ is a complex in $E^{7}, K_{a} \triangleleft K, f$ is a closed embedding of $\left(|K|,\left|K_{a}\right|\right)$ into $(M, \mathrm{Bd} M)$ where $M$ is a 3-manifold, and $s$ is a $0-, 1-$, or 2-simplex of $K$. If $s$ is a 1 -simplex suppose that $\mathrm{lk}(s, K)$ bas more than one component, each component of $\mathrm{lk}(s, K)$ is an arc, and $\mathrm{lk}(s, K) \cap K_{a}=\emptyset$ unless $s \in K_{a}$ in which case $\mathrm{lk}\left(s, K_{a}\right)$ is a 0 -sphere in $\mathrm{ik}(s, K)$. If $s$ is a vertex suppose that $\mathrm{lk}(s, K)$ bas some nonsimply connected component, each nondegenerate component of $\mathrm{lk}(s, K)$ is a punctured 2-sphere, and $\mathrm{lk}(s, K) \cap K_{a}=\varnothing$ unless $s \in K_{a}$ in which case $\mathrm{lk}\left(s, K_{a}\right)$ is a 1-sphere in the boundary of one of the components of $\mathrm{lk}(s, K)$.

Suppose $\mu \in C\left(M, f\left(\operatorname{st}^{\circ}(s, K)\right)\right)$ and $O$ is an open convex set in $E^{7}$ containing st $(s, K)$.

Then there are complexes $L_{a}<L$ in $E^{7}$ and there is a pwl map $r: L \rightarrow K$ such that $\left(L, L_{a}, r\right)$ reduces $\left(K, K_{a}\right)$ at $s$ and $r^{-1}(s t(s, K)) \subseteq O$, there is a closed 
embedding $g$ of $\left(|L|,\left|L_{a}\right|\right)$ into $(M, \mathrm{Bd} M)$, and there is a special map $\pi$ for $(M, f, g, r, \mu)$ which is the identity on $\mathrm{Bd} M$.

Proof. If $s$ is a 2-simplex the conclusion follows easily from Corollary 6.2. For the remaining two cases let $k$ be equal to the difference between the total number of boundary components of the nondegenerate components of $\mathrm{lk}(s, K)$ and the number of nondegenerate components of $\mathrm{lk}(s, K)$. Use Lemma 2.1 to find $\mu_{1} \in C\left(M, \mu^{-1}(0, \infty)\right)$ so that $\left(M, \mu / k, \mu_{1}, k+1\right)$ has Property S. Set $\left(L(0), L_{a}(0)\right)$ $=\left(K, K_{a}\right), g_{0}=f$, and $r_{0}=I: L(0) \rightarrow L(0)$. Let $M^{\prime}$ denote the sum of $M$ and $(\mathrm{Bd} M) \times[0,1]$ with the identification $x=(x, 0)$.

We consider two cases:

Case I. $s$ is a vertex. We define inductively a sequence of complex pairs $\left(L(i), L_{a}(i)\right)$, a sequence of pwl maps $r_{i}$, a sequence of embeddings $g_{i}:(|L(i)|$, $\left.\left|L_{a}(i)\right|\right) \rightarrow(M, \mathrm{Bd} M)$, and a sequence of special maps $\pi_{i}$ for $\left(M, g_{i-1}, g_{i}, r_{i}, \mu_{1}\right)$ which are the identity on $\mathrm{Bd} M$ so that each $\left(L(i), L_{\boldsymbol{a}}(i), r_{i}\right)$ is an elementary reduction of $\left(L(i-1), L_{\boldsymbol{a}}(i-1)\right)$ at a pair $\left(s_{i}, S_{i}\right)$ where $S_{i}<\operatorname{lk}(s, K), r_{1} \cdots$ $r_{i-1}\left(s_{i}\right)=s$, and $\left(r_{1} \cdots r_{i-1}\right)^{-1}(s t(s, K)) \subseteq O$.

Suppose that for some $1 \leq j<k$ the appropriate items have been defined for all $i<j$. Then for some vertex $s_{j}$ in $\left(r_{0} \cdots r_{j-1}\right)^{-1}(s)$ and some component $R_{j}$ of $\mathrm{lk}\left(s_{j}, L(j-1)\right), R_{j}$ has two boundary components. Choose $R_{j}$ so that if $s_{j} \epsilon$ $L_{a}(j-1)$ then $\operatorname{lk}\left(s_{j}, L_{a}(j-1)\right)<\dot{R}_{j}$. An invariance of domain argument can be used to show that this is always possible. Let $S_{j}$ be a 1 -sphere in $\dot{R}_{j}$ which misses $L_{a}(j-1)$. Notice that $S_{j}<\operatorname{lk}(s, K)$. Use [8, Lemma 2.1] to find a connected open set $W$ in $M^{\prime}$ containing $f\left(\right.$ Int $\left.s_{j} * S_{j}\right)$ so that $W-f\left(s_{j} * S_{j}\right)$ has two components $-W_{0}$ and $W_{1}, W_{0} \subseteq$ Int $M, C l W_{1}$ contains a neighborhood of $g_{j-1}\left(\right.$ Int $\left.s_{j} * S_{j}\right)$ in $g_{j-1}\left(s_{j} * R_{j}\right)$, and for each component $R$ of $\mathrm{Ik}\left(s_{j}, L(j-1)\right)$ one of $W_{0} \cap g_{j-1}\left(s_{j} * R\right)$ and $w_{1} \cap g_{j-1}\left(s_{j} * R\right)$ is empty. Let $R_{j 1}, \cdots, R_{j q}$, $\cdots, R_{j m}$ be an indexing of the components of $1 \mathrm{k}\left(s_{j}, L(j-1)\right)$ such that $g_{j-1}\left(s_{j} * R_{j i}\right) \cap W_{1} \neq \varnothing \Leftrightarrow i \leq q$.

Let $u_{j}$ be a point of $\left(E^{7}-L(j-1)\right) \cap O$ which is joinable to every finite subcomplex of $L(j-1)$. Let $L(j)$ denote the complex $\left[|L(j-1)|-s t^{\circ}\left(s_{j}, L(j-1)\right)\right]$ $\cup \bigcup_{i \leq q} s_{j} * R_{j i} \cup \bigcup_{i>q} u_{j} * R_{j i} \cup s_{j} * u_{j} * S_{j}$, and set $L_{a}(j)=L_{a}(j-1)$. Let $r_{j}$ denote the simplicial map from $L(j)$ to $L(j-1)$ which sends $u_{j}$ to $s_{j}$ and leaves every other vertex fixed. Then $\left(L(j), L_{a}(j), r_{j}\right)$ is an elementary reduction of $\left(L(j-1), L_{a}(j-1)\right)$ at $\left(s_{j}, S_{j}\right)$. Lemma 5.1 shows that $r_{j}$ is equivalent to a projection on $\left|s_{j} * u_{j} * s_{j}\right|-\left|s_{j}\right|$, so from Corollary 6.2 there is a closed embedding $g_{j}:\left(|L(j)|,\left|L_{a}(j)\right|\right) \rightarrow(M, \mathrm{Bd} M)$ and there is a special map $\pi_{j}$ for $\left(M, g_{j}, g_{j-1}\right.$, $\left.r_{j}, \mu_{1}\right)$.

After $k$ steps, the process terminates since for every vertex $v$ in $\left(r_{k} \cdots r_{1}\right)^{-1}(s)$ and for every nondegenerate component $R$ of $\mathrm{lk}(v, L(k)), R$ is a disk. Set 
$\left(L, L_{a}\right)=\left(L(k), L_{a}(k)\right), g=g_{k^{\prime}} r=r_{k} \cdots r_{1}$, and $\pi=\pi_{k} \cdots \pi_{1}$. Now $\left(L, L_{a^{\prime}} r\right)$ reduces $\left(K, K_{a}\right)$ at $s$, and from Lemmas 2.1 and $2.2, \pi$ is a special map for $(M, f, g, r, \mu)$.

Case II. $s$ is a 1-simplex. The items $L, L_{a}, r, g$, and $\pi$ can be obtained by an iterative construction analogous to the one in the preceding case. We show how to define an open set $W$ and a triple $\left(L(1), L_{a}(1), r_{1}\right)$ to be used in Corollary 6.2 to get the process started, and we leave all other details to the reader.

From an invariance of domain argument, if $s \in K_{a}$, then the two components of $\mathrm{lk}\left(s, K_{a}\right)$ belong to different components of $\mathrm{lk}(s, K)$. It follows then from [8, Lemma 2.1] that there are among the components of $\mathrm{lk}(s, K)$ two- $R_{1}$ and $R_{2}$; there are vertices $u \in \dot{R}_{1}-K_{a}$ and $v \in \dot{R}_{2}-K_{a}$, and there is a connected open set $W$ in $M^{\prime}$ containing $f(\operatorname{Int}(u * s \cup v * s))$ so that $W-f(u * s \cup v * s)$ has two components $-W_{0}$ and $W_{1}$ with $W_{0} \subseteq$ Int $M-f(|K|)$. For the complex $L(1)$ choose a point $w \in O$ which is joinable to each finite subcomplex of $K$ and set $L(1)=$ $K \cup s * w *(u \cup v)$ and $L_{a}(1)=K_{a}$. Define $r_{1}$ to be a retraction which is affine on each simplex of $L(1)$ and sends $w$ to $b(s)$.

After $(k-1)$ steps the link of $s$ is a 1 -cell and a reduction of $\left(K, K_{a}\right)$ at $s$ is achieved.

7. Neighborhoods for pinched 3-manifolds. Call a polyhedron $P$ a pinched 3-manifold if it fails to be a three manifold at no more than a discrete set of points and at each of the se points $P$ is locally equivalent to a cone over a finite collection of disjoint disks. In this section we rework McMillan's proofs from [21] to show how to find canonical neighborhoods for embeddings of polyhedra which are sums of pinched manifolds and graphs.

Lemma 7.1. Suppose $P$ is a polybedral 3-manifold, $P_{a}$ is either a polybedral surface in $\mathrm{Bd} P$ or the empty set, and $f$ is a closed embedding of $\left(P, P_{a}\right)$ into $(M, \mathrm{Bd} M)$ such that $f(P)$ is locally tame at $f\left(P_{a}\right)$. Set $P_{b}=(\operatorname{Bd} P)-\operatorname{Int} P_{a}$.

Suppose $\mu \in C(M, f(P))$.

Then there is a pwl homeomorphism $g$ of $\left(P, P_{a}\right)$ into $(M, \mathrm{Bd} M)$ and there is a discrete collection $\left\{C_{i}\right\}$ of polybedral 3-cells in $P$ with each $C_{i} \cap \mathrm{Bd} P$ a disk $D_{i}$ in Int $P_{b}$ so that

(1) $g(P)$ is tame and $g \mu$-approximates $f$,

(2) each $g\left(D_{i}\right)$ and each $f\left(C_{i}\right)$ is a $\mu$-set,

(3) $f\left(P-\bigcup\left(C_{i}-\operatorname{Fr} C_{i}\right)\right) \subseteq g\left(P-P_{b}\right)$, and

(4) for each $i, f(P) \cap g\left(D_{i}\right) \subseteq f\left(C_{i}\right)$.

Proof. Choose $\mu_{1} \in C(M, f(P))$ so that $\left(M, \mu / 2, \mu_{1}, 2\right)$ has Property S. Substitute $\left(M \rightarrow M, P_{b} \rightarrow S, \mathrm{Bd} P_{b} \rightarrow R, f \rightarrow f, \mu_{1} f \rightarrow \mu\right)$ in [9, Theorem 9.1] to get an associated $\nu \in C\left(P_{b}, P_{b}\right)$. Extend $\nu f^{-1}$ to $\mu_{2} \in C(M)$ so that $\mu_{2} \leq \mu_{1}$. 
From Lemma 2.4 there is a proper collar $\phi: P_{b} \times[0,1] \rightarrow P$ so that $f \phi(y \times[0,1])$ is a $\mu_{2} / 6$-set $\left(y \in P_{b}\right)$. From [1], [2], [22] we can suppose that $f \phi$ is locally pwl on $P_{b} \times(0,1]$.

From [18, Theorem 4] and [1], [2], [22] there is a homeomorphism $f_{0}:\left(P_{b}, \operatorname{Bd} P_{b}\right)$ $\rightarrow(M, \mathrm{Bd} M)$ which $\mu_{2} / 2$-approximates $f \mid P_{b}$ and there is a discrete collection of polyhedral disks $\left\{D_{i}\right\}$ in Int $P_{b}$ (discrete in Int $P_{b}$ ) so that $(1) f_{0}(x)=f(x)$ $\left(x \in \mathrm{Bd} P_{b}\right)$, (2) $f_{0}$ is locally pwl on Int $P_{b}$, (3) each $f\left(D_{i}\right) \cup f_{0}\left(D_{i}\right)$ is a $\mu_{2} / 2$ set, (4) $f\left(P_{b}\right) \cap f_{0}\left(\right.$ Int $\left.P_{b}\right) \subseteq U\left(f\left(\right.\right.$ Int $\left.D_{i}\right) \cap f_{0}\left(\right.$ Int $\left.\left.D_{i}\right)\right)$, and $(5) f(P) \cap f_{0}\left(P_{b}\right) \subseteq$ $f \phi\left(P_{b} \times[0,1 / 2)\right)$. For each $D_{i}$ set $C_{i}=\phi\left(D_{i} \times[0,1 / 2]\right)$. We suppose that (6) each $f_{0}\left(D_{i}\right)$ is in general position with respect to the open polyhedron $f\left(\left(\operatorname{Bd} C_{i}\right)-D_{i}\right)$. Since $f(P)$ is locally tame on $f\left(P_{a}\right)$ the proof of Theorem 4 of [18] shows that $f_{0}$ can be chosen so that $\left\{D_{i}\right\}$ is discrete not only in Int $P_{b}$ but also in $P_{b}$. Thus by pushing off $f(P)$ near $f\left(\mathrm{Bd} P_{b}\right)$ it is easy to define a pwl homeomorphism $f_{1}$ of $\left(P_{b}, \mathrm{Bd} P_{b}\right)$ into $(M, \mathrm{Bd} M)$ which has properties (2)-(6) when $f_{0}$ is replaced by $f_{1}$ and which has the additional property (7) that $f_{1}\left(\mathrm{Bd} P_{b}\right) \cap f(P)=\emptyset$.

For each $D_{i}, f\left(\operatorname{Fr} C_{i}\right) \cap f_{1}\left(D_{i}\right)$ separates $f_{1}\left(D_{i}\right) \cap f(P)$ from $f_{1}\left(D_{i}\right) \cap$ $f\left(P-C_{i}\right)$ in $f_{1}\left(D_{i}\right)$. Thus by cutting along components of $f_{1}\left(D_{i}\right) \cap f\left(\operatorname{Fr} C_{i}\right)$, sewing in new disks from $f\left(\operatorname{Fr} C_{i}\right)$, and then pushing the adjusted portions of $f_{1}\left(D_{i}\right)$ into $f\left(\right.$ Int $C_{i}$ ) for each $D_{i}$, we get a pwl homeomorphism $f_{2}$ of $P_{b}$ into $M$ which agrees with $f_{1}$ on the complement of $f_{2}^{-1}\left(U\right.$ Int $\left.C_{i}\right)$ so that each $f_{2}\left(D_{i}\right) \cap$ $f(P) \subseteq f\left(\right.$ Int $C_{i} \cup$ Int $\left.D_{i}\right)$. Since each $f\left(C_{i}\right)$ is a $\mu_{2} / 2$-set it follows that $f_{2}$ is a $\mu_{2} / 2$-approximation to $f$.

Define $f_{3}: P_{b} \rightarrow M$ by $f_{3}(x)=f \phi(x, 1)$. Since $f_{3}$ is also a $\mu_{2} / 2$-approximation to $f$ our application of [9] provides a pwl homeomorphism $b$ of $\left(P_{b} \times[0,1]\right.$, $\left.\left(\mathrm{Bd} P_{b}\right) \times[0,1]\right)$ into $(M, \mathrm{Bd} M)$ such that $b(y, 0)=f_{2}(y), b(y, 1)=f_{3}(y)$, and $b(y \times[0,1])$ is a $\mu_{1} f(y)$-set for each $y \in P_{b}$.

Define a homeomorphism $g_{0}$ of $P$ into $M$ by $g_{0} \mid P$ - image $\phi=f \mid P-$ image $\phi$ and $g_{0}=b \phi^{-1}$ on $\phi\left(P_{b} \times[0,1]\right)$. If $x=\phi(y, t)$ then $\rho\left(f(x), g_{0}(x)\right) \leq$ $\rho(f(x), f(y))+\rho\left(f(y), g_{0}(x)\right) \leq \mu_{2}(f(x)) / 6+\mu_{1}(f(y)) \leq \mu_{2}(f(x)) / 6+\mu(f(x)) / 2 \leq$ $(2 / 3) \mu(f(x))$. Thus by using [1], [2], [22] to take a close pwl approximation to $g_{0}$ which agrees with $g_{0}$ on $P_{b}$ and takes $P$ onto $g_{0}(P)$ we get a $g$ for which conditions (1)-(4) in the conclusion of the lemma are satisfied.

Theorem 7.2. Suppose $P$ is a polybedral 3-manifold, $P_{a}$ is either a polybedral surface in $\mathrm{Bd} P$ or the empty set, $M$ is a pwl 3-manifold, and $f$ is a closed embedding of $\left(P, P_{a}\right)$ into $(M, \mathrm{Bd} M)$. Suppose $\mu \in C(M, f(P))$.

Then there is a pwl homeomorphism $g$ of $\left(P, P_{a}\right)$ into $(M, \mathrm{Bd} M)$ and there is a discrete collection of polybedral cubes-with-handles $\left\{H_{i}\right\}$ in $M$ such that

(1) $g$ is a $\mu$-approximation to $f$,

(2) each $H_{i}$ is a $\mu$-set in Int $M$, 
(3) each $H_{i} \cap \mathrm{g}(P)$ is a disk in $\mathrm{g}(\mathrm{Bd} P)$, and

(4) $g(P) \cup \cup H_{i}$ is a closed neighborbood of $f(P)$.

Proof. The proof is divided into two cases:

Case I. $f(P)$ is locally tame on $f\left(P_{a}\right)$. This case follows from Lemma 7.1 exactly as Theorem 1 of [21] follows from Lemmas 1 and 2 there.

Case II. General case. Only Case I of the theorem is used in the remainder of this paper; thus we can use Theorem 9.1 in obtaining the general case. We omit the $\mu$ 's and just sketch the proof.

Define a proper collar $\lambda: P_{b} \times[0,1] \rightarrow P$ on $P_{b}$ in $P$ so that $f \lambda$ is locally pwl on $P_{b} \times(0,1)$, and apply Theorem 9.1 to get a pwl embedding $b:\left(P_{b} \times[0,1 / 2]\right.$, $\left.\left(\mathrm{Bd} P_{b}\right) \times[0,1 / 2]\right) \rightarrow(M, \mathrm{Bd} M)$ and a discrete collection of polyhedral cubes-withhandles $\left\{G_{i}\right\}$ so that $b\left(P_{b} \times[0,1 / 2]\right) \cup \cup G_{i}$ contains a neighborhood of $f\left(P_{b}\right)$ and $b\left(P_{b} \times 1 / 2\right)$ lies between $b\left(P_{b} \times 0\right)$ and $f \lambda\left(P_{b} \times 1\right)$. Then apply [9] as in the proof of the preceding lemma to extend $b$ to a pwl embedding of $\left(P_{b} \times[0,1]\right.$, $\left.\left(\mathrm{Bd} P_{b}\right) \times[0,1]\right)$ into $(M, \mathrm{Bd} M)$ so that $b(y, 1)=f \lambda(y, 1)$ for $y \in P_{b}$. Define $g^{\prime}: P \rightarrow M$ by $g^{\prime}=f$ on $P$ - image $\lambda$ and $g^{\prime} \lambda(y, t)=b(y, t)$. Take a pwl approximation to $g^{\prime}$ which agrees with $g^{\prime}$ on $P_{b}$ to get $g^{\prime}$. The $H_{i}$ 's are obtained from the $G_{i}$ 's by throwing out those which are contained in $g$ (Int $P$ ).

Theorem 7.3. Suppose $Q_{a}=P_{a}<P<Q$ are polybedra such that $P$ is a pinched 3-manifold, $P_{a}$ is either a surface which bas a 3-manifold neighborbood in $P$ or is the empty set, $\mathrm{Cl}(Q-P) \cap P_{a}=\emptyset$, and dimension $(Q-P) \leq 1$.

Suppose $f$ is a closed embedding of $\left(Q, Q_{a}\right)$ into $(M, \mathrm{Bd} M)$ such that $f(P)$ is locally tame on $f\left(P_{a}\right)$ and $\mu \in C(M, f(Q))$.

Then there is a canonical neighborbood system for $(M, Q, f, \mu)$.

Proof. Let $\mu_{1} \in C(M, f(Q))$ correspond to $M, f(Q), \mu$, and $k=5$ in Corollary 3.5. Let $\left(L, K, K_{a}, \phi\right)$ be a triangulation of $\left(Q, P, P_{a}\right)$ with $K, K_{a} \triangleleft L$ such that, for each $t \in L, f \phi(N(t, L))$ is a $\mu_{1}$-set. Let $\left\{v_{i}\right\}$ denote the vertices of $K$ whose links are not connected, and let $\left\{u_{k}\right\}$ denote the vertices of $L_{0}-K_{0}$. Further, let $\left\{\sigma_{j}\right\}$ denote the 1 -simplexes of $L-K$. Let $L^{2}$ denote the second barycentric subdivision of $L$.

Let $\{O(t): t \in L\}$ be a collection of orientable, open $\mu_{1}$-sets such that (1) for each $t, f \phi\left(N\left(t, L^{2}\right)\right) \subseteq O(t)$, (2) $O(s) \cap O(t)=\emptyset$ if $s \cap t=\varnothing$, (3) $O(t) \subseteq O(s)$ if $t<s$, and (4) $O(t) \cap \mathrm{Bd} M=\varnothing$ if $t \cap K_{a}=\varnothing$. For each $u_{k}$ let $B\left(u_{k}\right)$ be a pwl 3 -cell in $O\left(u_{k}\right)$ such that (5) $f \phi\left(u_{k}\right) \in$ Int $B\left(u_{k}\right)$ and (6) $f(Q) \cap B d B\left(u_{k}\right)$ is totally disconnected.

Set $P(0)=\phi\left(\bigcup v_{i}\right), P(1)=P-P(0)$, and $P(2)=\operatorname{Cl}\left(P-\bigcup \phi\left(N\left(v_{i}, K^{2}\right)\right)\right)$.

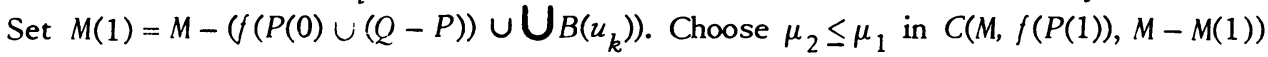
so that (7) for each $t \in L, \sup \left\{\mu_{2}(x): x \in O(t)\right\}<d\left(f \phi\left(N\left(v_{i}, L^{2}\right)\right), M-O(t)\right)$. 
Apply Theorem 7.2 for the substitution $\left(M(1) \rightarrow M, P(1) \rightarrow P, f \rightarrow f, \mu_{2} \rightarrow \mu\right)$ to get a pwl embedding $g_{1}$ of $\left(P(1), P_{a}\right)$ into $(M(1), \mathrm{Bd} M)$ and a discrete collection of polyhedral cubes-with-handles $\left\{G_{k}\right\}$ in $M(1)$ so that the four conditions are satisfied in the conclusion of the theorem. We suppose that any $G_{k}$ is thrown out if it does not intersect $f(P)$. From (7) if $G_{k} \cap f \phi\left(N\left(v_{i}, L^{2}\right)\right) \neq \emptyset$ then $G_{k} \subseteq$ $O\left(v_{i}\right)$. We suppose that $g_{1}$ has been adjusted so that (8) each $g_{1} \phi\left(\left|1 \mathrm{k}\left(v_{i}, K^{2}\right)\right|\right)$ misses $\bigcup G_{k}$ and (9) each $g_{1} \phi\left(N\left(v_{i}, K^{2}\right)\right)$ fails to intersect $f(P(2))$. Extend $g_{1}$ to all of $Q$ by setting $g_{1}=f$ on $Q-P(1)$.

In each $O\left(v_{i}\right)$ let $C\left(v_{i}\right)$ be a compact, polyhedral 3-manifold such that (10) $C\left(v_{i}\right) \cap g_{1}(P)=\dot{g}_{1} \phi\left(N\left(v_{i}, K^{2}\right)\right),(11)\left(\mathrm{Bd} C\left(v_{i}\right)\right) \cap g_{1}(P)=g_{1} \phi\left(\left|1 \mathrm{k}\left(v_{i}, K^{2}\right)\right|\right)$, (12) for each $G_{k}, G_{k} \subseteq C\left(v_{i}\right)$ or $G_{k} \cap C\left(v_{i}\right)=\varnothing$ accordingly as $G_{k} \cap g_{1} \phi\left(N\left(v_{i}, K^{2}\right)\right) \neq$ $\emptyset$ or $G_{k} \cap g_{1} \phi\left(N\left(v_{i}, K^{2}\right)\right)=\emptyset$, (13) $N(1)=g_{1}(P) \cup \cup C\left(v_{i}\right) \cup \cup G_{k}$ contains a neighborhood of $f(P)$, and (14) (Bd $\left.C\left(v_{i}\right)\right) \cap f(Q-P)$ is totally disconnected.

Each $D\left(v_{i}\right)=g_{1}(P) \cap \mathrm{Bd} C\left(v_{i}\right)$ is a collection of mutually exclusive disks; thus from [20, Lemma 1] there is a finite collection $\left\{A_{i j}\right\}$ of mutually exclusive polyhedral arcs in $C\left(v_{i}\right)-D\left(v_{i}\right)$ which span $\mathrm{Bd} C\left(v_{i}\right)$ so that the closure of $C\left(v_{i}\right)$ minus a regular neighborhood of $\bigcup_{j} A_{i j}$ in $C\left(v_{i}\right)-D\left(v_{i}\right)$ is a cube-with-handles. We can suppose each $\mathrm{Bd} A_{i j}$ misses $f(Q)$. Each st $\left(v_{i}, L^{2}\right) \searrow^{s} v_{i} * V_{i} \cup$ $\mathrm{lk}\left(v_{i}, L^{2}\right)$ where $V_{i}$ is a complex having one vertex in each component of $\mathrm{lk}\left(v_{i}, L^{2}\right)$. From [20, Corollary 1.1] there is a pwl homeomorphism $b_{1}$ of $M$ onto itself which is the identity on $(M-N(1)) \cup\left(M-\bigcup O\left(v_{i}\right)\right)$ so that (15) each $b_{1}\left(A_{i j}\right) \cap f(Q)=\varnothing$ and (16) $b_{1} \mid G_{k} \neq I \Rightarrow G_{k} \subseteq O\left(v_{i}\right)$ for some $i$. Condition (16) can be obtained by requiring that $b_{1}$ be the identity on all $G_{k}$ 's which fail to intersect any $f \phi\left(N\left(v_{i}, L^{2}\right)\right)$. Set $g_{2}=b_{1} g_{1}$.

In each $b_{1}\left(C\left(v_{i}\right)\right)$ subtract a regular neighborhood of $b_{1}\left(U_{j} A_{i j}\right)$ which misses both $g_{2} \phi\left(\left|\mathrm{lk}\left(v_{i}, K^{2}\right)\right|\right)$ and $f(Q)$ and take the closure of what remains to get a polyhedral cube-with-handles $E\left(v_{i}\right)$. By (8), (11), and (14), $E\left(v_{i}\right)=B\left(v_{i}\right) \cup F\left(v_{i}\right)$ where (17) $B\left(v_{i}\right)$ is a pwl 3-cell, (18) $F\left(v_{i}\right)$ is a polyhedral cube-with-handles missing $g_{2}(P(2))$, (19) $B\left(v_{i}\right) \cap F\left(v_{i}\right)$ is a disk, and (20) $f(Q-P) \cap \operatorname{Bd} E\left(v_{i}\right) \subseteq$ $\left(\operatorname{Bd} B\left(v_{i}\right)\right)-F\left(v_{i}\right)$. Set $N(2)=g_{2}(P(2)) \cup \cup B\left(v_{i}\right)$. Note that $N(3)=N(2) \cup$ $\bigcup\left\{G_{k}: G_{k} \cap C\left(v_{i}\right)=\varnothing\right.$ for every $\left.v_{i}\right\} \cup \bigcup F\left(v_{i}\right)$ contains a neighborhood of $f(P)$ in $M$.

Using the same constructions as in the preceding two paragraphs we find a collection $\left\{E\left(\sigma_{j}\right)\right\}$ of mutually exclusive polyhedral cubes-with-handles in Int $M$ such that (21) $N(3) \cup \cup B\left(u_{k}\right) \cup \cup E\left(\sigma_{j}\right)$ contains a neighborhood of $f(Q)$ in $M$, and for each $\sigma_{j}$, (22) $E\left(\sigma_{j}\right) \subseteq O\left(\sigma_{j}\right),(23) N(3) \cap E\left(\sigma_{j}\right)$ is a pair of disks in $\operatorname{Bd}\left(N(3) \cup \cup B\left(u_{k}\right)\right)$, one in each $B(t)$ for which $t<\sigma_{j}$, (24) $E\left(\sigma_{j}\right) \cap F\left(v_{i}\right)=\varnothing$ for every $v_{i}$, and (25) $E\left(\sigma_{j}\right)=B\left(\sigma_{j}\right) \cup F\left(\sigma_{j}\right)$ where $B\left(\sigma_{j}\right)$ is a pwl 3-cell, $F\left(\sigma_{j}\right)$ is

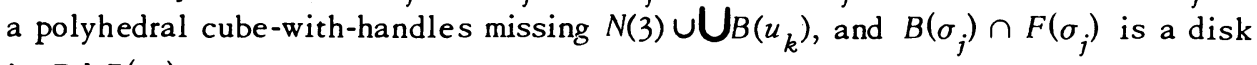
in $\mathrm{Bd} B\left(\sigma_{j}\right)$.

Let $S_{1}$ denote the polyhedral surface $(\operatorname{Bd} P(2))-\left(\phi\left(U\left|\mathrm{k}\left(v_{i}, K^{2}\right)\right|\right) \cup\right.$ 
Int $P_{a}$ ), and set $S=\mathrm{Cl} S_{1}$. From Lemma 2.4 there is a proper pwl collar $\lambda: S \times$ $[0,2] \rightarrow P(2)$ such that (26) for each simplex $t$ of $K$ with $\phi(t) \cap S=\varnothing$, $g_{2}^{-1}(O(t))$ contains $\lambda((S \cap \phi(t)) \times[0,2])$.

Define a pwl homeomorphism $g$ of $\left(Q, Q_{a}\right)$ into $(M, \mathrm{Bd} M)$ as follows: On $P(2)$ - image $\lambda$ set $g=g_{2}$. On image $\lambda$ set $g \lambda(y, t)=g_{2} \lambda\left(y, \theta\left(\begin{array}{ll}0 & 2 \\ 1 & 2\end{array}\right)(t)\right)$. Extend $g$ tn $Q$ by using the cone structures on the cells $B(t)$ to have $g$ take (27) each $\phi\left(N\left(v_{i}, L^{2}\right)\right)$ conewise into $B\left(v_{i}\right),(28)$ each $\phi\left(N\left(u_{k}, L^{2}\right)\right)$ conewise into $B\left(u_{k}\right)$, and (29) each $\phi\left(N\left(b\left(\sigma_{j}\right), L^{2}\right)\right)$ conewise into $B\left(\sigma_{j}\right)$ so that if $u_{k}$ or $v_{i}<\sigma_{j}$ then $g \phi\left(b\left(u_{k} * b\left(\sigma_{j}\right)\right)\right)$ or $g \phi\left(b\left(v_{i} * b\left(\sigma_{j}\right)\right)\right)$ belongs to Int $\left(B\left(u_{k}\right) \cap B\left(\sigma_{j}\right)\right)$ or Int $\left(B\left(v_{i}\right) \cap B\left(\sigma_{j}\right)\right)$. From conditions on $g_{2}$ and the $B(t)$ 's and from condition (26), $g$ is a $\mu$-approximation to $f$.

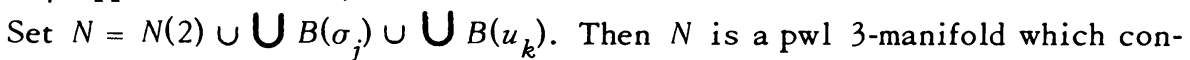
tains a neighborhood of $g(Q)$, and the sum of $N$ and $\operatorname{Cl}(N(3)-N(2)) \cup \cup F\left(\sigma_{j}\right)$ is a closed, polyhedral neighborhood of $f(Q)$. Let $(T, \psi)$ be a triangulation of $S$ such that, for each $t \in T, \lambda(\psi(N(t, T)) \times[0,1]) \subseteq O(s)$ for some $s \in L$. Let $T^{2}$ be the second barycentric subdivision of $T$. For $i=0, \cdots, 3$ set

$$
\begin{aligned}
& N_{i}=\mathrm{Cl}\left(N-\bigcup\left\{\lambda \left(\left(\psi\left(N\left(b(t), T^{2}\right) \cap S_{1}\right) \times[0,1)\right):\right.\right.\right. \\
& t\text { is a simplex of } \Gamma \text { of dimension } \geq 3-i\}) .
\end{aligned}
$$

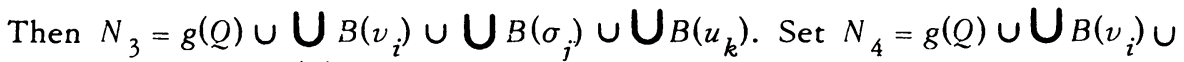
$\bigcup B\left(u_{k}\right)$ and $N_{5}=g(Q)$. Now $N=N_{0} \searrow N_{1} \searrow \cdots \searrow N_{5}$, and each of these collapses is a $\mu_{1}$-collapse which is admissible with respect to $B d M$. From our application of Corollary 3.5, $N$ is a $\mu$-regular neighborhood of $g(Q)$.

For the cubes-with-handles $\left\{H_{i}\right\}$ take $\left\{F\left(v_{i}\right)\right\} \cup\left\{F\left(\sigma_{j}\right)\right\} \cup\left\{G_{k}: G_{k} \cap C\left(v_{i}\right)=\varnothing\right.$ for every $\left.v_{i}\right\}$. Then $\left(N, g,\left\{H_{i}\right\}\right)$ is a canonical neighborhood system for $(M, Q, f, \mu)$.

8. Existence and uniqueness of canonical neighborhoods.

Theorem 8.1. Suppose $Q_{a}<Q$ are polybedra, $M$ is a pwl 3-manifold, $f$ is a closed embedding of $\left(Q, Q_{a}\right)$ into $(M, \mathrm{Bd} M)$, and $\mu \in C(M, f(Q))$.

Then there is a canonical neigbborbood system $\left(g, N,\left\{H_{i}\right\}\right)$ for $(M, Q, f, \mu)$.

Proof. The central idea in the proof is to make repeated applications of the splitting theorems in $\$ 6$ to transform $f$ into an embedding of a polyhedron for which Theorem 7.3 applies and then to use properties of special maps and reducing systems to recover a canonical neighborhood system for $f(Q)$. We will construct a tower of polyhedra and maps indicated in Diagram 8.1. This tower will be commutative with respect to solid lines. The $Q$ 's denote polyhedra, the i's pwl inclusions or embeddings, the r's pwl maps, the f's topological embeddings, the $g$ 's pwl approximations to the $f$ 's, the $\pi$ 's special maps, and $H$ a pwl homeomorphism approximating $\pi$. 


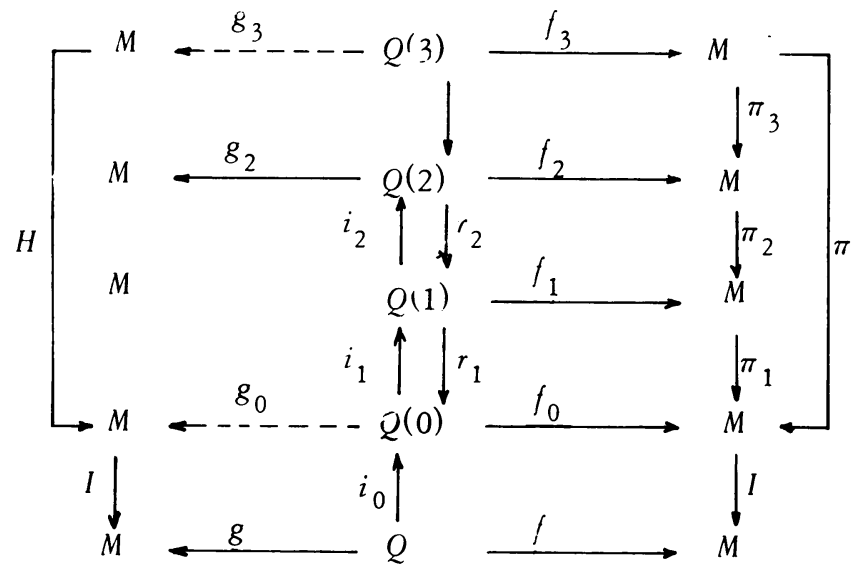

Diagram 8.1

Step 1. First story of tower. Let $\mu_{1} \in C(M, f(Q))$ correspond to $\mu_{1}$ in Corollary 3.5 for the substitution $(M \rightarrow M, f(Q) \rightarrow Y, \mu \rightarrow \mu, 10 \rightarrow k)$. Because $f\left(Q_{a}\right)$ is tame in $\mathrm{Bd} M$ there is some pwl structure on $\mathrm{Bd} M$ making $f \mid Q_{a} \mathrm{pwl}$. Let $i_{0}: Q \rightarrow E^{7}$ and $j_{0}: \mathrm{Bd} M \rightarrow E^{7}$ be closed embeddings of $Q$ and $\mathrm{Bd} M$ onto polyhedra in $E^{7}$ such that (1) $i_{0}^{-1} j_{0}(\mathrm{Bd} M)=Q_{a}$, (2) $j_{0}^{-1} i_{0}\left|Q_{a}=f\right| Q_{a}$, and (3) $i_{0}$ is pwl. By Lemma 3.6 there is a regular neighborhood $Q_{a}(0)$ of $i_{0}\left(Q_{a}\right)$ in $j_{0}(\mathrm{Bd} M)$ such that $j_{0}^{-1}\left(Q_{a}(0)\right)$ is a $\mu_{1}$-regular neighborhood of $f\left(Q_{a}\right)$ with an appropriate pwl structure put on $\operatorname{Bd} M$. Set $Q(0)=i_{0}(Q) \cup Q_{a}(0), P_{a}(0)=Q_{a}(0)$, and let $P(0)$ denote the set of points of $Q(0)$ where the local dimension of $Q(0)$ exceeds one. Define $f_{0}: Q(0) \rightarrow M$ by $f_{0}=f i_{0}^{-1}$ on $i_{0}(Q)$ and $f_{0}=j_{0}^{-1}$ on $Q_{a}(0)$.

Choose $\mu_{2} \in C\left(M, f_{0}(Q(0))\right)$ so that $\mu_{2} \leq \mu_{1}$ and so that if $f_{0}^{\prime}:\left(Q(0), Q_{a}(0)\right)$ $\rightarrow(M, \mathrm{Bd} M)$ is a pwl $\mu_{2}$-approximation to $f_{0}$ then $f_{0}^{\prime}\left(Q_{a}(0)\right)$ is $\mu_{1}$-regular neighborhood of $f_{0}^{\prime} i_{0}\left(Q_{a}\right)$ in Bd M. Theorem 7.2 of [10] together with the two dimensional approximation theorems in [1], [2], [22] can be used to show that such a $\mu_{2}$ exists. Use Lemma 2.1 to find $\mu_{3}$ and $\mu_{4} \in C\left(M, f_{0}(Q(0))\right)$ so that (4) $\left(M, \mu_{2} / 6,2 \mu_{3}, 2\right)$ and (5) $\left(M, \mu_{3} / 3, \mu_{4}, 4\right)$ have Property S. Let $L(0)$ be a rectiline ar complex in $E^{7}$ such that (6) $|L(0)|=Q(0)$, (7) there are full subcomplexes $K(0)$ and $K_{a}(0)$ whose carriers are respectively $P(0)$ and $P_{a}(0)$, and (8) for each $t \in L(0), f_{0}(N(t, L(0)))$ is a $\mu_{4}$-set. Choose open $\mu_{4}$-sets $O(t)$ for the simplexes $t$ of $L(0)$ such that, for each $t \in L(0), f_{0}(N(t, L(0))) \subseteq O(t)$ and $s<t \Rightarrow O(s) \subseteq$ $O(t)$.

Step 2. Second story of tower. Let $\left\{\Delta_{i}\right\}$ denote the 2-simplexes of $L(0)$ whose links are empty. Let $\left\{y_{i}\right\}$ be a collection of mutually exclusive points in $E^{7}-Q(0)$ so that (9) every finite subset of $\left\{y_{i}\right\}$ is joinable to every finite subcomplex of $L(0)$ and (10) $\rho\left(y_{i}, b\left(\Delta_{i}\right)\right)<1 / i$ for each $i$. Set $K(1)=K(0) \cup \cup y_{i} * \Delta_{i}$, $L(1)=K(1) \cup L(0), K_{a}(1)=K_{a}(0), P(1)=|K(1)|, Q(1)=|L(1)|$, and $Q_{a}(1)$ $=P_{a}(1)=P_{a}(0)$. Let $i_{1}$ denote the inclusion of $Q(0)$ into $Q(1)$, and let $r_{1}$ denote 
the retraction of $Q(1)$ onto $Q(0)$ which is affine on each simplex of $L(1)$ and sends each $y_{i}$ to $b\left(\Delta_{i}\right)$.

Let $\left\{U\left(\Delta_{i}\right)\right\}$ be a collection of mutually exclusive open sets in $M$ such that (11) each $U\left(\Delta_{i}\right) \cap f_{0}(Q(0))=f_{0}\left(\right.$ Int $\left.\Delta_{i}\right)$ and (12) $U\left(\Delta_{i}\right) \subseteq O(t)$ provided $\Delta_{i} \cap t \neq \emptyset$. By applying Theorem 6.3 for each $\Delta_{i}$ we find that there is a closed embedding $f_{1}:\left(Q(1), Q_{a}(1)\right) \rightarrow(M, \mathrm{Bd} M)$ and there is a special map $\pi_{1}$ for $\left(M, f_{0}, f_{1}, r_{1}, \mu_{4}\right)$ which is the identity on $\left(M-\bigcup U\left(\Delta_{i}\right)\right) \cup \operatorname{Bd} M$.

Step 3. Third story of tower. Let $\left\{\sigma_{j}\right\}$ denote the 1 -simplexes of $K(1)$ whose links are not connected. Set $L(2,1)=\left(L(1), L_{1}(1)\right)^{2}$ the relative second barycentric subdivision, and let $K(2,1)$ and $K_{a}(2,1)$ denote the appropriate subcomplexes corresponding to $K(1)$ and $K_{a}(1)$. Let $\left\{U\left(\sigma_{j}\right)\right\}$ be a collection of mutually exclusive open sets in $M$ such that (13) each $U\left(\sigma_{j}\right) \cap f_{1}(Q(1))=f_{1}\left(\operatorname{st}^{\circ}\left(\sigma_{j}, L(2,1)\right)\right)$, and (14) each $U\left(\sigma_{j}\right) \subseteq O(t)$ provided $\sigma_{j} \cap t \neq \emptyset$.

Repeated applications of Theorem 6.3 show that there is a complex pair $\left(L(2), L_{a}(2)\right)$ and there is a pwl retraction $r_{2}:|L(2)| \rightarrow Q(1)$ such that (L(2), $\left.L_{a}(2), r_{2}\right)$ reduces $\left(L(2,1), L_{a}(2,1)\right)$ at the 1 -simplexes $\left\{\sigma_{j}\right\}$, and there is an embedding $f_{2}$ of $\left(|L(2)|,\left|L_{a}(2)\right|\right)$ into $(M, \mathrm{Bd} M)$ and there is a special map $\pi_{2}$ for $\left(M, f_{1}, f_{2}, r_{2}, \mu_{4}\right)$ which is the identity on $(\operatorname{Bd} M) \cup\left(M-\cup U\left(\sigma_{j}\right)\right)$. Set $Q(2)=$ $|L(2)|, Q_{a}(2)=P_{a}(2)=P_{a}(1)$, let $P(2)$ denote the set of points of $Q(2)$ where the local dimension exceeds one, and let $K(2)$ and $K_{a}(2)$ denote the subcomplexes of $L(2)$ whose carriers are $P(2)$ and $P_{a}(2)$. Let $i_{2}$ denote the inclusion of $Q(1)$ into $Q(2)$.

Step 4. Fourth story of tower. Let $L^{2}(2)$ be a second derived subdivision of $L(2)$ inducing a subdivision $L^{\prime}(0)$ of $L(0)$ for which $r_{1} r_{2}: L^{2}(2) \rightarrow L^{\prime}(0)$ is simplicial. Let $\left\{v_{k}\right\}$ denote the vertices $v$ of $K(2)$ for which $\mathrm{lk}(v, L(2))$ fails to be a 2-cell or a 2 -sphere, and let $\left\{u_{k}\right\}$ denote the vertices of $L_{0}(2)-K_{0}(2)$. Both these sets are contained in $L_{0}(0)$. From invariance of domain it follows that each component of each $\mathrm{lk}\left(v_{k}, L^{2}(2)\right)$ is either a point or a punctured 2-sphere. Let $\left\{U\left(v_{k}\right)\right\}$ be mutually exclusive open sets in $M$ such that (15) each $f_{2}(Q(2)) \cap U\left(v_{k}\right)$ $=f_{2}\left(s t^{\circ}\left(v_{k}\right), L^{2}(2)\right)$, and (16) each $U\left(v_{k}\right) \subseteq O\left(v_{k}\right)$.

An application of Lemma 6.3 like the previous one shows that there are (17) complexes and polyhedra $L(3), L_{a}(3), Q(3)=|L(3)|$, and $Q_{a}(3)=\left|L_{a}(3)\right|$, (18) a simplicial map $r_{3}$ from $L(3)$ to $L^{2}(2)$ such that $\left(L(3), L_{a}(3), r_{3}\right)$ reduces $\left(L^{2}(2)\right.$, $\left.L_{a}^{2}(2)\right)$ at the vertices $\left\{v_{k}\right\},(19)$ an embedding $f_{3}:\left(Q(3), Q_{a}(3)\right) \rightarrow(M, \operatorname{Bd} M)$ such that each $f_{3}^{-1}\left(\mathrm{Cl} U\left(v_{k}\right)\right)=r_{3}^{-1}\left(N\left(v_{k}, L^{2}(2)\right)\right)$, and (20) a special map $\pi_{3}$ for $\left(M, f_{2}, f_{3}, r_{3}, \mu_{4}\right)$ which is the identity on $(\mathrm{Bd} M) \cup\left(M-\cup U\left(v_{k}\right)\right)$. Let $P(3)$ denote the subpolyhedron of $2(3)$ consisting of the points where the local dimension of $Q(3)$ exceeds one, set $P_{a}(3)=Q_{a}(3)$, and let $K(3)$ and $K_{a}(3)$ denote the full subcomplexes of $L(3)$ whose carriers are $P(3)$ and $P_{a}(3)$.

Notice that $Q(3)$ is the sum of a graph and the pinched 3 -manifold $P(3)$, and 
that $K(3)$ fails to be a combinatorial 3-manifold only at certain vertices $w$ in $\left(K_{0}(3)-K_{a 0}(3)\right) \cap r_{3}^{-1}\left(\left\{v_{k}\right\}\right)$ where at each such $w$ every component of $\mathrm{lk}(w, K(3))$ is a disk. Notice also that (21) each $\left(r_{1} r_{2} r_{3}\right)^{-1}\left(\left|1 \mathrm{k}\left(v_{k}, L^{\prime}(0)\right)\right|\right)$ is a surface because $r_{1}{ }_{2}: L^{2}(2) \rightarrow L^{\prime}(0)$ is simplicial.

Step 5. A neighborbood for $f(Q)$. Set $\pi=\pi_{1} \pi_{2} \pi_{3}$ and $r=r_{1} r_{2}$. For each simplex $t$ of $L(3), \pi f_{3}(N(t, L(3))) \subseteq O(s)$ provided $\mathrm{rr}_{3}(t) \subseteq s$. From Theorem 7.3 there is a canonical neighborhood system $\left(g_{3}, N(3),\left\{G_{i}\right\}\right)$ for $\left(M, Q(3), f_{3}, \mu_{4}\right)$. From the proof of Theorem 7.3 we may suppose that (22) for each $t \in L(3)$, $\pi g_{3}(N(t, L(3))) \subseteq O(s)$ provided $\mathrm{rr}_{3}(t) \subseteq s$ and (23) there are mutually exclusive pwl 3-manifolds $B(t)$ in the sets (Int $M) \cap \pi^{-1}(O(t))$ for $t \in\left\{u_{k}\right\} \cup\left\{v_{k}\right\}$ so that each $B(t)$ is a regular neighborhood of $g_{3} r^{-1}\left(N\left(b(t), L^{2}(2)\right)\right)$ modulo $g_{3}(Q(3)-N(b(t), L(3)))$ in $M$ and $N(3)=N_{0}(3) \searrow \cdots \searrow N_{4}(3)=g_{3}(Q(3)) \cup \cup_{B\left(v_{k}\right) \cup}$ $B\left(u_{k}\right)$ by a sequence of four $\mu_{4}$-collapses admissible with respect to $\mathrm{Bd} M$ where each component of each $\mathrm{Cl}\left(N_{i-1}(3)-N_{i}(3)\right)$ is contained in some $\pi^{-1}(O(s))$. Condition (21) enables us to achieve condition (23). From Lemma 5.2 and [16] we see that each $B(t)$ is a pwl 3-cell.

Now $g_{3} r^{-1} \mid g_{3}^{-1}\left(\mathrm{Cl}\left(M-\bigcup_{B}\left(v_{k}\right)\right)\right)$ is a pwl embedding.. Use the cone structures on the $B\left(v_{k}\right)$ 's to extend this restriction to a pwl embedding $g_{2}$ of $(Q(2)$, $\left.Q_{a}(2)\right)$ into $(M, \mathrm{Bd} M)$ so that each $g_{2}^{-1}\left(\right.$ Int $\left.B\left(v_{k}\right)\right)=\mathrm{st}^{\circ}\left(v_{k}, L^{2}(2)\right)$. Set $g_{0}=$ $g_{2} i_{2}{ }^{i}{ }_{1}$. Set $N_{5}(3)=g_{2}(Q(2))$. Let $L^{\prime \prime \prime}(0)$ be the second barycentric subdivision of $L^{\prime}(0)$, the subdivision mentioned at the beginning of step 4. For each $t \in L^{\prime}(0)$, $g_{2} r^{-1}\left(N\left(b(t), L^{\prime \prime \prime}(0)\right)\right) \subseteq \pi^{-1}(O(s))$ for some $s$. Set $N_{5+i}(3)=g_{0}(Q(0)) \cup$ $\bigcup\left\{g_{2} r^{-1}\left(N\left(b(t), L^{\prime \prime \prime}(0)\right)\right): t\right.$ is a simplex of dimension $\leq 2-i$ in $\left.L^{\prime}(0)\right\}$. Then $N_{4}(3) \searrow N_{5}(3) \searrow \ldots \searrow N_{8}(3)=g_{0}(Q(0))$, each of these collapses is admissible with respect to $\mathrm{Bd} M$, and each component of each $\mathrm{Cl}\left(N_{i-1}(3)-N_{i}(3)\right)$ is contained in some $\pi^{-1}(O(s))$.

Because $\pi_{0}$ is the composition of three special $\mu_{4}$-maps it is a special $\mu_{3}$ map. Further, $\pi\left(N(3) \cup \cup G_{i}\right)$ contains a neighborhood of $f(Q)$. Thus from [1], [2], [22] there is a pwl $2 \mu_{3}$-homeomorphism $H$ of $M$ onto itself which approximates $\pi$ so closely that (24) $H\left(N(3) \cup \cup G_{i}\right.$ ) contains a neighborhood of $f(Q)$, and (25) each component of each $H\left(\mathrm{Cl}\left(N_{i-1}(3)-N(3)\right)\right)$ is contained in $O(s)$ for some $s$. Set $g=H g_{0} i_{0}, N=H(N(3))$, and $\left\{H_{i}\right\}=\left\{H\left(G_{i}\right)\right\}$. We claim that $\left(g, N,\left\{H_{i}\right\}\right)$ is a canonical neighborhood system for $(M, Q, f, \mu)$. First $g_{0}$ is a $\mu_{4}$-approximation to $f_{0}$ and $H$ is a $2 \mu_{3}$-map so $H_{0}$ is a $\mu_{2}$-approximation to $f_{0}$. Thus $g$ is a $\mu$-approximation to $f$. Furthermore by Lemma 3.6 and the conditions on $\mu_{2}$, if we set $N_{i}=H\left(N_{i}(3)\right)$ then $N=N_{0} \searrow \cdots \searrow N_{8} \searrow N_{9} \searrow N_{10}=g(Q)$ by a sequence of $10 \mu_{1}$-collapses which are admissible with respect to $\mathrm{Bd} M$. From Corollary $3.5, N$ is a $\mu$-regular neighborhood of $g(Q)$. Similar conside rations show that each $H_{i}$ is a $\mu$-set. 
Except for the distribution of cubes-with-handles canonical neighborhoods are unique. This statement is given meaning by the following theorem on $\mu$-regular ne ighborhoods.

Theorem 8.2. Suppose $Q_{a}<Q$ are polybedra, $M$ is a pwl 3-manifold, $f$ is a closed embedding of $\left(Q, Q_{a}\right)$ into $(M, \mathrm{Bd} M)$, and $\mu \in C(M, f(Q))$.

There is a $\mu_{1} \in C(M, f(Q))$ such that if $g_{1}$ and $g_{2}$ are pwl embeddings of $\left(Q, Q_{a}\right)$ into $(M, \mathrm{Bd} M)$ which $\mu_{1}$-approximate $f$, and if $N_{1}$ and $N_{2}$ are $\mu_{1}$-regular neighborboods of $g_{1}(Q)$ and $g_{2}(Q)$ respectively, then there is a pwl $\mu$-homeomorphism $b$ of $\left(N_{1}, N_{1} \cap \mathrm{Bd} M\right)$ onto $\left(N_{2}, N_{2} \cap \mathrm{Bd} M\right)$ which extends $g_{2} g_{1}^{-1}$.

Proof. Use Lemma 2.1 and [11, Theorem 3.1$]$ to find $\mu_{2}, \mu_{3}$, and $\mu_{1} \epsilon$ $C(M, f(Q))$ so that $\left(M, \mu / 3,2 \mu_{2}, 4\right),\left(M, \mu_{2} / 2,2 \mu_{3}, 3\right)$, and $\left(M, \mu_{3}, \mu_{1}, 2\right)$ have Property $\mathrm{S}$, and so that if $f^{\prime}$ and $f^{\prime \prime}$ are pwl $\mu_{1}$-approximations to $f$ which take $\left(Q, Q_{a}\right)$ into $(M, \mathrm{Bd} M)$, then there is a pwl extension of $f^{\prime \prime}\left(f^{\prime}\right)^{-1}$ which takes a polyhedral neighborhood pair $\left(R^{\prime}, R^{\prime} \cap \operatorname{Bd} M\right)$ of $\left(f^{\prime}(Q), f^{\prime}\left(Q_{a}\right)\right)$ onto a polyhedral neighborhood pair $\left(R^{\prime \prime}, R^{\prime \prime} \cap \operatorname{Bd} M\right)$ of ( $f^{\prime \prime}(Q), f^{\prime \prime}\left(Q_{a}\right)$ ).

Let $g_{1}, g_{2}, N_{1}$, and $N_{2}$ be given as in the hypothesis. Let $b_{0}$ be the promised pwl extension of $g_{2} g_{1}^{-1}$ which takes a neighborhood pair $\left(R_{1}, R_{1} \cap \mathrm{Bd} M\right)$ onto a neighborhood pair $\left(R_{2}, R_{2} \cap \mathrm{Bd} M\right)$. Note that $g_{2} g_{1}^{-1}$ is a $\mu_{3}$-map; thus by Lemma 3.6 there are $\mu_{1}$-regular neighborhoods $N_{1}^{\prime}$ of $g_{1}(Q)$ in $R_{1}$ and $N_{2}^{\prime}=$ $b_{0}\left(N_{1}^{\prime}\right)$ of $g_{2}(Q)$ in $R_{2}$ such that $b_{0} \mid N_{1}^{\prime}$ is a $2 \mu_{3}$-map. Furthermore by Lemma 3.1 there are pwl $\mu_{2}$-homeomorphisms $b_{1}$ of $\left(N_{1}, N_{1} \cap \mathrm{Bd} M\right)$ onto $\left(N_{1}^{0}, N_{1}^{\prime} \cap \mathrm{Bd} M\right)$ and $b_{2}$ of $\left(N_{2}^{\prime}, N_{2}^{\prime} \cap \mathrm{Bd} M\right)$ onto $\left(N_{2}, N_{2} \cap \mathrm{Bd} M\right)$ which are the identity on $f_{1}(Q)$ and $f_{2}(Q)$ respectively. Thus $b_{2} b_{0} b_{1}$ is a $\mu$-map and a pwl extension of $g_{2}^{-1} g_{1}$ which takes $\left(N_{1}, N_{1} \cap \mathrm{Bd} M\right)$ onto $\left(N_{2}, N_{2} \cap \mathrm{Bd} M\right)$.

9. Canonical neighborhoods for surfaces. In this section we exhibit an alternate characterization of canonical neighborhoods for surfaces which is more in the spirit of McMillan's work.

Theorem 9.1. Suppose $S$ is a polybedral surface, $R \subseteq \mathrm{Bd} S$ is either a poly. bedral 1-manifold or the empty set, $M$ is a pwl 3-manifold, and $f$ is a closed embedding of $(S, R)$ into $(M, \mathrm{Bd} M)$. Suppose that $\mu \in C(M, f(S))$.

If $f(($ Int $S) \cup($ Int $R)$ ) is two sided in some open subset of $M$, then there is a pwl bomeomorphism $g$ of $(S \times[0,1], R \times[0,1])$ into $(M, \mathrm{Bd} M)$ and there is a discrete collection of polybedral cubes-with-bandles $\left\{H_{i}\right\}$ in $M$ such that

(1) for each $x \in S$, and each $t \in[0,1], \rho(f(x), g(x, t)) \leq \mu(f(x))$,

(2) eacb $H_{i}$ is a $\mu$-set in Int $M$ which intersects $g(S \times[0,1])$ in a disk, and

(3) $g(S \times[0,1]) \cup \cup H_{i}$ contains a neighborbood of $f(S)$.

Proof. From $\$ 8$ it is sufficient to show that for each $\mu \in C(M, f(Q))$ there is some pwl homeomorphism $g$ of $(S \times[0,1], R \times[0,1])$ into $(M, \mathrm{Bd} M)$ which 
satisfies condition (1) and there is some pwl embedding $f_{0}:(S, R) \rightarrow(M, \mathrm{Bd} M)$ which $\mu$-approximates $f$ so that $g(S \times[0,1])$ is a $\mu$-regular neighborhood of $f_{0}(S)$.

Let $\mu_{1}$ correspond to $M, \mu, f(Q)$, and $k=5$ in Corollary 3.5. Let $\mu_{2} \epsilon$ $C(M, f($ Int $S \cup \operatorname{Int} R), f((\mathrm{Bd} S)-R))$ be such that $\mu_{2} \leq \mu_{1}$. From Theorem 6.1 , the definition of special map, and [2], there is a locally pwl homeomorphism $g^{\prime}:$ ((Int $S$ $U$ Int $R) \times[0,1],($ Int $R) \times[0,1]) \rightarrow(M, \mathrm{Bd} M)$ so that, for each $y \in$ Int $S \cup$ Int $R$ and each $t \in[0,1], \rho\left(f(y), g^{\prime}(y, t)\right) \leq \mu_{2} f(y)$ and $g^{\prime}(y \times[0,1])$ is a $\mu_{2}$-set.

Let $\lambda:((\operatorname{Bd} S)-$ Int $R) \times[0,3] \rightarrow S$ be a proper pwl collar on (Bd $S)-$ Int $R$ in $S$ so small so that if $g: S \times[0,1] \rightarrow M$ is defined by $g \mid(S-$ image $\lambda) \times[0,1]$ $=g^{\prime} \mid(S$ - image $\lambda) \times[0,1]$ and $g(\lambda(y, s), t)=g^{\prime}\left(\lambda\left(y, \theta\left(\begin{array}{ll}0 & 3 \\ 1 & 3\end{array}\right)(s)\right), t\right)$, then each $g(y \times[0,1])$ is a $\mu_{1}$-set, for each $(y, t) \in S \times[0,1], \rho(g(y, t), f(y)) \leq \mu_{1}(f(y))$, and if $f_{0}$ denotes the pwl embedding $f_{0}(y)=g^{\prime}(y, 1 / 2)$ for $y \notin$ image $\lambda$ and $f_{0} \lambda(y, t)=g^{\prime}\left(\lambda\left(y, \theta\left(\begin{array}{ll}0 & 3 \\ 2 & 3\end{array}\right)(t)\right), 1 / 2\right)$, then $f_{0} \mu_{1}$-approximates $f$. Now $g(S \times[0,1]) \searrow$ $g(S \times 1 / 2)$ by a sequence of $3 \mu_{1}$-collapses admissible with respect to $\mathrm{Bd} M$ and $g(S \times 1 / 2) \succ f_{0}(S)$ by two $\mu_{1}$-collapses admissible with respect to $\mathrm{Bd} M$. These five collapses are essentially those introduced in the proofs of Theorems 3.6 and 7.3. From Corollary 3.5, $g(S \times[0,1])$ is a $\mu$-regular neighborhood of $f_{0}(S)$.

\section{REFERENCES}

1. R. H. Bing, Locally tame sets are tame, Ann of Math. (2) 59 (1954), 145-158. MR $15,816$.

2. - An alternative proof that 3-manifolds can be triangulated, Ann. of Math. (2) 69 (1959), 37-65. MR 20 \#7269.

3. - Pushing a 2-sphere into its complement, Michigan Math. J. 11(1964), 33-45. MR $28 \# 3408$.

4. - A surface is tame if its complement is 1-ULC, Trans. Amer. Math. Soc. 101 (1961), 294-305. MR 24 \#A1117.

5. - Improving the side approximation theorem, Trans. Amer. Math. Soc. 116 (1965), 511-525. MR 33 \#704.

6. M. Brown, Locally flat embeddings of topological manifolds, Ann. of Math. (2) 75 (1962), 331-341. MR 24 \#A3637.

7. M. M. Cohen, A general theory of relative regular neighborhoods, Trans. Amer. Math. Soc. 136 (1969), 189-229. MR 40 \#2052.

8. R. Craggs, Improving the intersection of polyhedra in 3-manifolds, Illinois J.

Math. 12 (1968), 567-586. MR 38 \#692.

9. - Building cartesian products of surfaces with $[0,1]$, Trans. Amer. Math.

Soc. 144 (1969), 391-425. MR 40 \#3553.

10. - Small ambient isotopies of a 3-manifold which transform one embedding of a polyhedron into another, Fund. Math. 68 (1970), 225-256.

11. - Extending small homeomorphisms between approximating polyhedra, Duke Math. J. 38 (1971), 161-168.

12. R. J. Daver man, A new proof for the Hosay-Lininger theorem about crumpled cubes, Proc. Amer. Math. Soc. 23 (1969), 52-54. MR 39 \#7578.

13. R. J. Daverman and W. T. Eaton, An equivalence for the embeddings of cells in a 3-manifold, Trans. Amer. Math. Soc. 145 (1969), 369-381. MR 40 \#3519.

14. J. Dugundji, Topology, Allyn and Bacon, Boston, Mass., 1966. MR $33 \# 1824$.

15. N. Hosay, The sum of a cube and a crumpled cube is $S^{3}$, Notices Amer. Math.

Soc. 11 (1964), 152. Abstract \#607-17. (Erratum.) 
16. J. F. P. Hudson and E. C. Zeeman, On regular neighborhoods, Proc. London Math. Soc. (3) 14 (1964), 719-745. MR 29 \#4063.

17. L. L. Lininger, Some results on crumpled cubes, Trans. Amer. Math. Soc. 118 (1965), 534-549. MR 31 \#2717.

18. F. M. Lister, Simplifying intersections of disks in Bing's side approximation theorem, Pacific J. Math. 22 (1967), 281-295. MR 35 \#7317.

19. J. Martin, Sewings of crumpled cubes which do not yield $S^{3}$, Topology Seminar (Wisconsin, 1965), Ann. of Math. Studies, no. 60, Princeton Univ. Press, Princeton, N. J., 1966 , pp. $57-59$.

20. D. R. McMillan, Jr., A criterion for cellularity in a manifold. II, Trans. Amer. Math. Soc. 126 (1967), 217-224. MR 34 \#8392.

21. - Neighborhoods of surfaces in 3-manifolds, Michigan Math. J. 14 (1967), 161-170. MR $35 \# 3643$.

22. E. E. Moise, Affine structures on 3-manifolds, VIII. Invariance of the knot types; local tame embedding, Ann. of Math. (2) 59 (1954), 159-170. MR 15, 889.

23. A. Scott, Infinite regular neighbourhoods, J. London Math. Soc. 42 (1967), 245253. MR $35 \# 3672$.

24. J. R. Stallings, Lectures on polyhedral topology, Tata Inst. Fund. Res. Lectures on Math., no. 43, Tata Institute of Fundamental Research, Bombay, 1967. MR 38 \#6605.

25. W. L. Voxman, On the shrinkability of decompositions of 3-manifolds, Notices Amer. Math. Soc. 15 (1968), 649-650. Abstract \#68T-497.

26. J.H. C. Whitehead, Simplicial spaces, nuclei and m-groups, Proc. London Math. Soc. 45 (1939), 243-327.

27. E. C. Zeeman, Seminar on combinatorial topology, Publ. Inst. Hautes Études Sci., Paris, 1963. (mimeographed notes).

DEPARTMENT OF MATHEMATICS, UNIVERSITY OF ILLINOIS, URBANA, ILLINOIS 61801 